\title{
Die Zukunft der evangelischen Kirche: Prognosen für Österreich sowie für das Burgenland, Kärnten und Wien
}

\author{
Anne Goujon und Claudia Reiter ${ }^{1}$ \\ (deutsche Übersetzung der englischen Fassung²)
}

Die Studie wurde von der Evangelischen Kirche A.B. in Österreich finanziell unterstützt.

\section{Executive Summary}

Die Stellung der evangelischen Kirche in der österreichischen Gesellschaft wurde in den letzten Jahrzehnten durch die Kräfte von Säkularisation und Migration erheblich beeinflusst. Die Zahl der Mitglieder ging deutlich zurück: von 447.00o Mitgliedern im Jahr 1971 auf 278.00o im Jahr 2018. Dieser Trend ist über alle Bundesländer hinweg zu erkennen, obwohl die Dimensionen in Wien stärker auffallen, wo sowohl eine größere Bereitschaft zum Austritt besteht als auch mehr internationale Migration stattfindet. So sank in der Hauptstadt die Zahl der evangelischen Mitglieder im Zeitraum 1971-2018 von 126.000 auf 47.000.

Mit Hilfe von Bevölkerungsprojektionen der Mitgliederzahlen der evangelischen Kirche untersuchen wir deren mögliche Zukunft in ganz Österreich und in drei einzelnen Bundesländern (Wien, Kärnten und Burgenland) im Zeitraum von 2018 bis 2048, wobei verschiedene Trends bei der Fertilität und auch beim Religionsaustritt berücksichtigt werden. Wir betrachten auch die Auswirkungen der unterschiedlichen Zusammensetzung der internationalen Migrationsströme einschließlich der Zugehörigkeit der Migrantinnen und Migranten zur evangelischen Kirche.

Wie unsere Ergebnisse zeigen, wird die evangelische Kirche - ohne kompensierende Maßnahmen - eindeutig weiter schrumpfen, sofern es ihr nicht gelingt, den Abwanderungsprozess ihrer Mitglieder zu stoppen. Die Prognosen belegen aber auch, dass Migrantinnen und Migranten, insbesondere solche aus dem mobilen Europa, eine potenzielle Mitgliederressource darstellen, die derzeit jedoch nicht optimal zu den Mitgliederzahlen in Österreich beiträgt. Nach dem Szenario TREND EUROPE, dem - aus heutiger Sicht - wahrscheinlichsten mit weiterhin rückläufigen Ein- und zunehmenden Austritten, würde die evangelische Bevölkerung in Österreich von 283.000 im Jahr 2018 auf 144.00o im Jahr 2048 sinken (-49\%). Auf der Ebene der drei Bundesländer verliefe der Rückgang in Wien von 47.000 auf $27.000(-42 \%$, von 47.000 auf 27.000) schneller als im Burgenland (-26\%, von 32.000 auf 24.000) und in Kärnten (-37\%, von 48.00o auf 30.000). Gemäß diesem Szenario würde die evangelische Bevölkerung in Österreich im Jahr 2048 1,5\% der Gesamtbevölkerung entsprechen (1,3\% in Wien, 7,3\% im Burgenland und 5,4\% in Kärnten). Andere Szenarien zeigen die potenziellen Auswirkungen der unterschiedlichen religiösen Zusammensetzung der Migrantinnen und Migranten sowie

\footnotetext{
${ }^{1}$ Wittgenstein Centre for Demography and Global Human Capital (IIASA, VID/ÖAW, Universität Wien).

${ }^{2}$ Englische Fassung „The future of the Protestant Church: Estimates for Austria and for the Provinces of Burgenland, Carinthia and Vienna" verfügbar unter: https://www.oeaw.ac.at/fileadmin/subsites/Institute/VID/PDF/Publications/Working_Papers/WP2020_ 2.pdf
} 
verschiedener Trends beim Austritt. Nur das optimistische LIFT-Szenario, unter dessen Annahmen sich die Austritte halbieren und sich mehr Zugewanderte in die evangelische Kirche einschreiben, kann den Aderlass stoppen und die Zahl der Mitglieder in Wien sogar erhöhen. 


\title{
Die Zukunft der evangelischen Kirche: Prognosen für Österreich sowie für das Burgenland, Kärnten und Wien
}

Anne Goujon und Claudia Reiter ${ }^{3}$

\begin{abstract}
In Anlehnung an den Bericht von Goujon, Jurasszovich und Potančoková (2017), der die Bevölkerung von Wien und Österreich nach Religionszugehörigkeit für den Zeitraum 20012016 schätzte, sowie an ein früheres Projekt von Fliegenschnee, Goujon und Lutz (2004), das sich mit der Zukunft der evangelischen Kirche bis 2031 beschäftigte, soll dieses Projekt anhand der neuesten Daten für ganz Österreich und die drei Bundesländer Burgenland, Kärnten und Wien aktualisiert werden. Ziel der vorliegenden Studie soll es sein, die Frage nach den möglichen Auswirkungen der Demographie (insbesondere Fertilität und Migration) und dem Trend zum Religionsaustritt auf die künftigen Mitgliederzahlen der evangelischen Kirche zu beantworten.
\end{abstract}

\section{Einleitung}

Evangelische Gemeinden gibt es seit mehreren Jahrhunderten als Minderheitsreligion in Österreich - das ja überwiegend römisch-katholisch geprägt ist. Seit den 1970er Jahren stehen beide christlichen Konfessionen vor wichtigen Veränderungen und zwar bedingt durch zwei Hauptkräfte: Säkularisierung und Migration.

Säkularisierung bezeichnet den Prozess, dass die Religion aufgrund von Modernisierung und Aufklärung allmählich die übergreifende Bedeutung verliert, die sie einst im Leben der Menschen besaß. Während Religion für die meisten Menschen immer noch zur kulturellen Identität gehört, ist sie heute mehr zu einem individuellen Attribut geworden. Die Säkularisierung hat zu einem Rückgang der Religiosität geführt, wobei weniger Menschen den Glauben praktizieren, in den sie hineingeboren wurden. Während diese Phänomene überall im globalen Norden zu beobachten sind, sind sie wesentlich sichtbarer - und jedenfalls messbarer - in Ländern mit Kirchensteuern zur Finanzierung der religiösen Organisationen, egal ob der Staat (wie in Deutschland) oder die Kirchen selbst (wie in Österreich) diese von ihren registrierten Mitgliedern einheben (Kirchensteuern oder -beiträge gibt es in Europa außerdem auch in Dänemark, Schweden, Finnland und der Schweiz). Der Säkularisierungstrend wird durch die nur geringe Vermittlung der Religion von Eltern auf ihre Kinder verstärkt, sodass auch die Zahl der getauften Kinder abnimmt.

Die Migration wirkte sich auch auf die den historischen Religionsgemeinschaften zugewiesene Stellung aus, indem sie das Gewicht anderer Religionen und damit die religiöse Vielfalt erhöhte. In den letzten dreißig Jahren hat die Zuwanderung nach Österreich durch den gestiegenen Arbeitskräftebedarf und auch infolge von Krisen in Nachbarländern wie etwa dem Jugoslawienkrieg und dem Fall der Berliner Mauer zugenommen. In jüngerer Zeit führten der im Jahr 2011 ausgebrochene Krieg in Syrien und andere Konflikte in Afghanistan, Irak, Eritrea sowie die bittere Armut in weiteren Ländern dazu, dass mehr Menschen nach alternativen Lebensmöglichkeiten suchten. Migrantinnen und Migranten haben über einen Zeitraum von

\footnotetext{
${ }^{3}$ Wittgenstein Centre for Demography and Global Human Capital (IIASA, VID/ÖAW, Universität Wien).
} 
mehreren Jahrzehnten religiöse Traditionen nach Österreich mitgebracht, die in der Bevölkerung vorher seltener waren, insbesondere den Islam und das orthodoxe Christentum.

Die Veränderungen aufgrund von Säkularisierung und Migration werden durch die Alterung der Bevölkerung - eine demographische Entwicklung, die nicht nur in Österreich, sondern in vielen Ländern des Globalen Norden zu beobachten ist - noch weiter verstärkt. Während ein längeres Leben einerseits mehr potenzielle Mitgliedsjahre in der Evangelischen Kirche bedeuten kann, führt die Alterung der Gesellschaft andererseits auch dazu, dass weniger Kinder geboren werden und somit ein geringeres Potenzial an neuen Mitgliedern vorhanden ist.

Sowohl die Säkularisierungstendenzen als auch die religiöse Diversifikation, die die heutige Glaubenslandschaft Österreichs prägen, lassen sich beziffern, die Zukunft ist jedoch weitgehend unbekannt. Die Datenbank der evangelischen Kirche zeigt einen deutlichen Rückgang der Zahl ihrer Mitglieder. ${ }^{4}$ Diese verringerte sich von 447.000 im Jahr 1971 auf 278.00o im Jahr 2018, also um 38\% (-1\% durchschnittliche jährliche Wachstumsrate), während zugleich die österreichische Bevölkerung von 7,9 auf 8,8 Millionen anstieg, also ein Plus von 11\% (+0,3\% durchschnittliche jährliche Wachstumsrate). Diese beunruhigende Statistik liefert den Ausgangspunkt für Prognosen, die darauf abzielen, die zukünftigen Mitgliederzahlen der evangelischen Kirche von 2018 bis 2048 für Österreich und drei Bundesländer (Burgenland, Kärnten und Wien) auf Grundlage mehrerer Szenarien zu quantifizieren.

Dieser Bericht unterscheidet sich von bereits bestehenden Prognosen zur Zukunft der Evangelischen Kirche. Während Goujon et al. (2017) den Fokus auf Religionszugehörigkeit (selbst-definiertes Zugehörigkeitsgefühl) gelegt haben, konzentrieren wir uns in diesem Bericht auf zukünftige Entwicklungen der tatsächlichen Mitgliederzahlen. Das war auch Schwerpunkt der Prognosen, welche unter Fliegenschnee et al. (2004) erstellt wurden, die in diesem Bericht mit aktuellen Daten $\mathrm{zu}$ demographischen Trends und MitgliederEntwicklungen aktualisiert werden.

Im Anschluss an die Einführung betrachten wir in Teil 2 die Veränderungen, die es seit 2001 in der evangelischen Bevölkerung gegeben hat, und zwar anhand von Volkszählungsdaten und Mitgliederzahlen der evangelischen Kirche. In Teil 3 wird kurz auf frühere Arbeiten eingegangen, die ebenfalls die evangelische Bevölkerung prognostiziert haben. Unsere Daten, Methoden und Szenarien für die Projektion der Mitgliederzahl der evangelischen Kirche bis 2048 sind in Teil 4 angeführt. Im letzten Teil werden die Ergebnisse der diversen Szenarien aufbereitet und diskutiert.

\section{Mitgliederzahlen der evangelischen Kirche seit 2001}

Wie eingangs erwähnt, haben sich Struktur und Größe der evangelischen Kirche in Österreich in den letzten Jahren stark verändert. Waren 2001 noch rund 376.00o Einwohner Österreichs Mitglieder der evangelischen Kirche (laut Volkszählung), so ist diese Zahl seither deutlich zurückgegangen: nur noch rund 278.00o Menschen in Österreich gehören heute der evangelischen Kirche an - trotz anhaltendem Bevölkerungswachstum in Österreich. Damit ist

\footnotetext{
${ }^{4}$ Die Datenbank enthält nur Mitglieder der Evangelischen Kirche A.B. (Augsburgisches Bekenntnis); Mitglieder der reformierten Tradition der Evangelischen Kirche H.B. (= Helvetischen, d.h. Schweizerischen Bekenntnisses) sind hier nicht erfasst. Die Mehrheit der Evangelischen in Österreich (ca. 96\%) gehört der Evangelischen Kirche A.B. an.
} 
auch der Anteil der evangelischen Kirchenmitglieder an der Gesamtbevölkerung deutlich gesunken - von 4,4 Prozent im Jahr 2001 auf nur noch 3,2 Prozent im Jahr 2018.

Abbildung 1 zeigt die Entwicklung der evangelischen Kirchengemeinde in Österreich seit 2001 und veranschaulicht den deutlichen Abwärtstrend, den die evangelische Kirche in der Vergangenheit sowohl in Bezug auf die absoluten Mitgliederzahlen als auch auf deren relativen Anteil an der Gesamtbevölkerung verzeichnet hat.

Abb. 1. Mitgliederzahlen der Evangelischen Kirche A.B., Österreich, 2001-2018

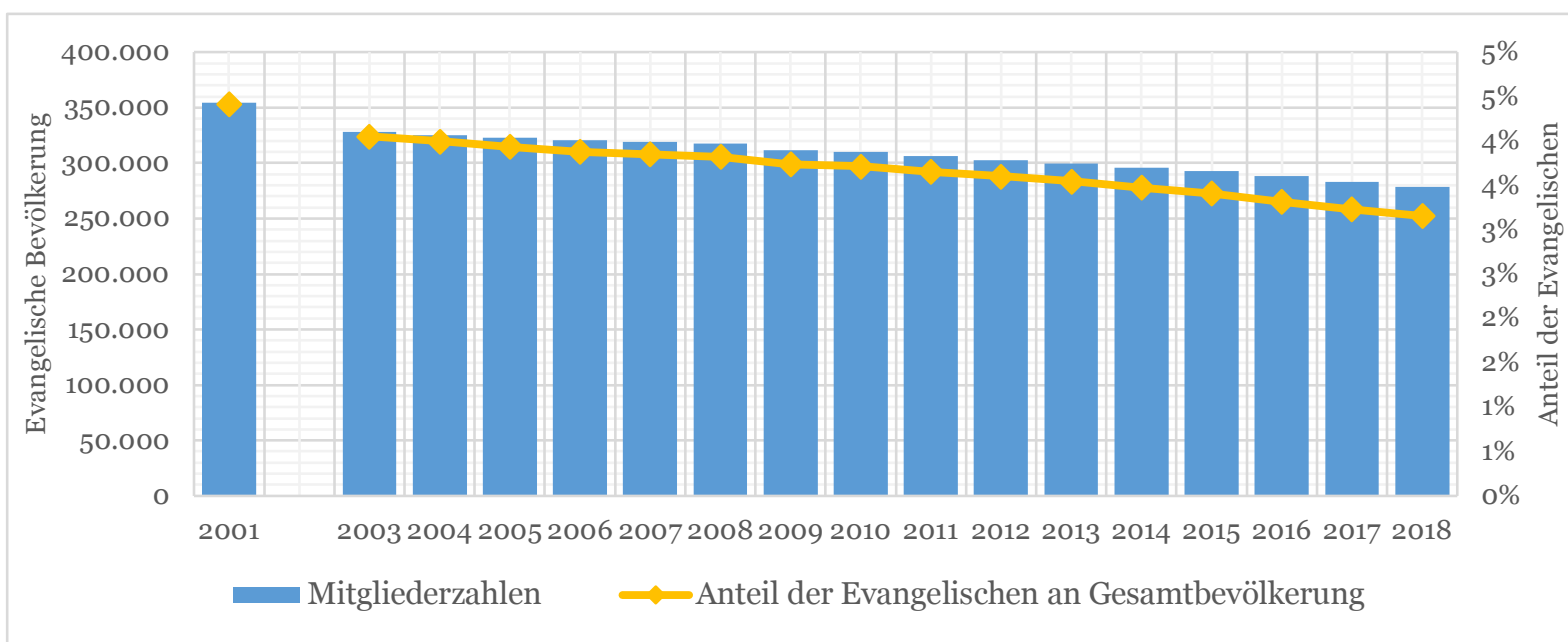

Quellen: 2001: Statistik Österreich (Volkszählung); 2003-2018: Amtsblatt für die evangelische Kirche in Österreich

Ähnliche Trends sind in den Bundesländern Burgenland, Kärnten und Wien zu beobachten, wenn auch in unterschiedlichem Tempo und Ausmaß. Abbildung 2 zeigt die jährliche Entwicklung der evangelischen Mitgliederzahlen in Prozent der Zahl aus 2003 (2003=100\%). Wie die Grafik zeigt, verzeichnete die Hauptstadt Wien einen stärkeren und schnelleren Mitgliederverlust als die beiden vorwiegend ländlich geprägten Bundesländer Burgenland und Kärnten. Dennoch war der deutliche und kontinuierliche Mitgliederrückgang ein genereller Trend der evangelischen Kirche in ganz Österreich (siehe auch Abbildung 4). Bemerkenswert ist, dass in den letzteren zwei Bundesländern die Zahl der Mitglieder in etwa konstant blieb, von 1971 bis 2001 rund 58.000 in Kärnten und 38.000 im Burgenland, während die Mitgliederzahl in Wien bereits im gleichen Zeitraum von 126.000 auf 72.000 zurückging (siehe Abbildung 5). 
Abb. 2. Jährliche Entwicklung der Zahl der evangelischen Gemeindemitglieder (A.B.) $(2003=100), 2003-2018$

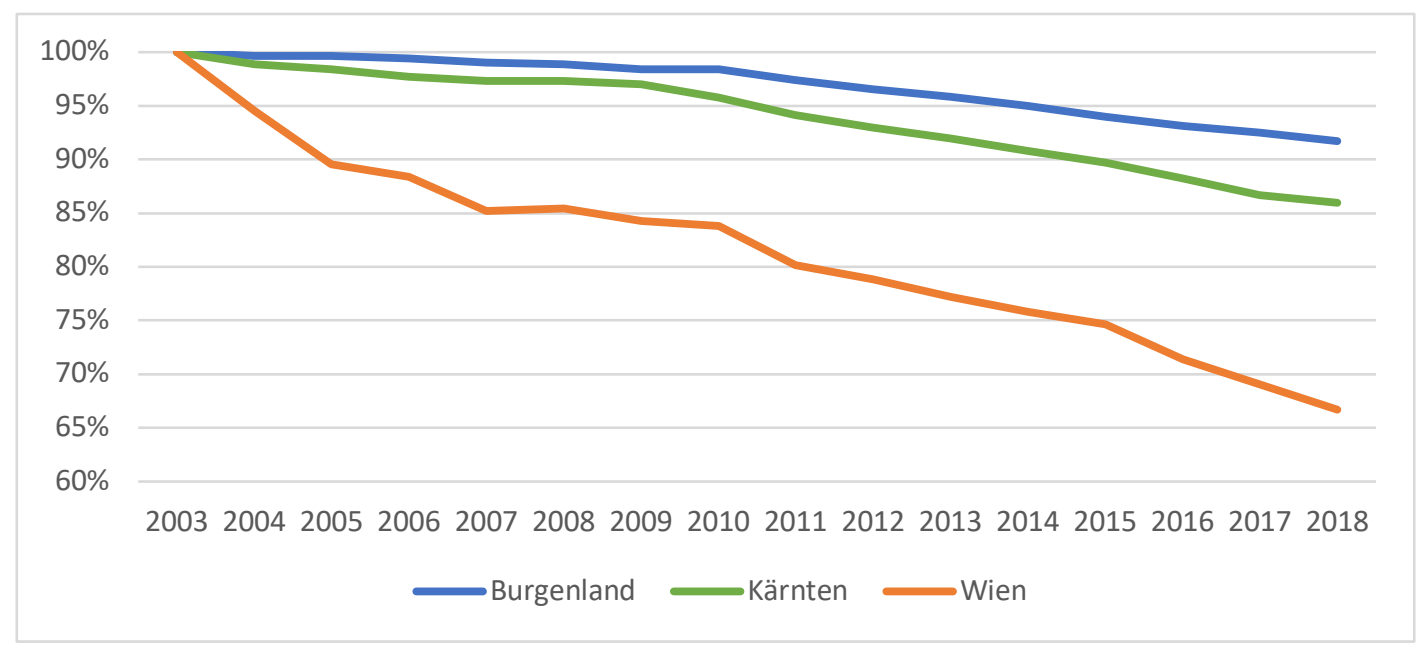

Quelle: Amtsblatt für die evangelische Kirche in Österreich 2004-2019

Der Hauptgrund für diese bemerkenswerten Veränderungen im Verlauf der letzten fünfzehn Jahre liegt in der zunehmenden Zahl von Kirchenaustritten. In Abbildung 3 sind die NettoAustrittsraten (Differenz zwischen Ein- und Austritte dividiert durch die Anzahl der evangelischen Kirchenmitglieder pro 1.000 Einwohner) für ganz Österreich sowie das Burgenland, Kärnten und Wien dargestellt. Wie sich zeigt, war diese Rate für alle Jahre und Regionen negativ, d.h. es verließen jeweils mehr Menschen die evangelische Kirche als in sie eintraten. Tempo und Ausmaß dieses Trends variieren jedoch wieder von Bundesland zu Bundesland, wobei Wien während des gesamten Zeitraums an der Spitze steht.

Abb. 3. Netto-Austrittsraten für die Evangelische Kirche A.B. (2003=100), 2003-2018

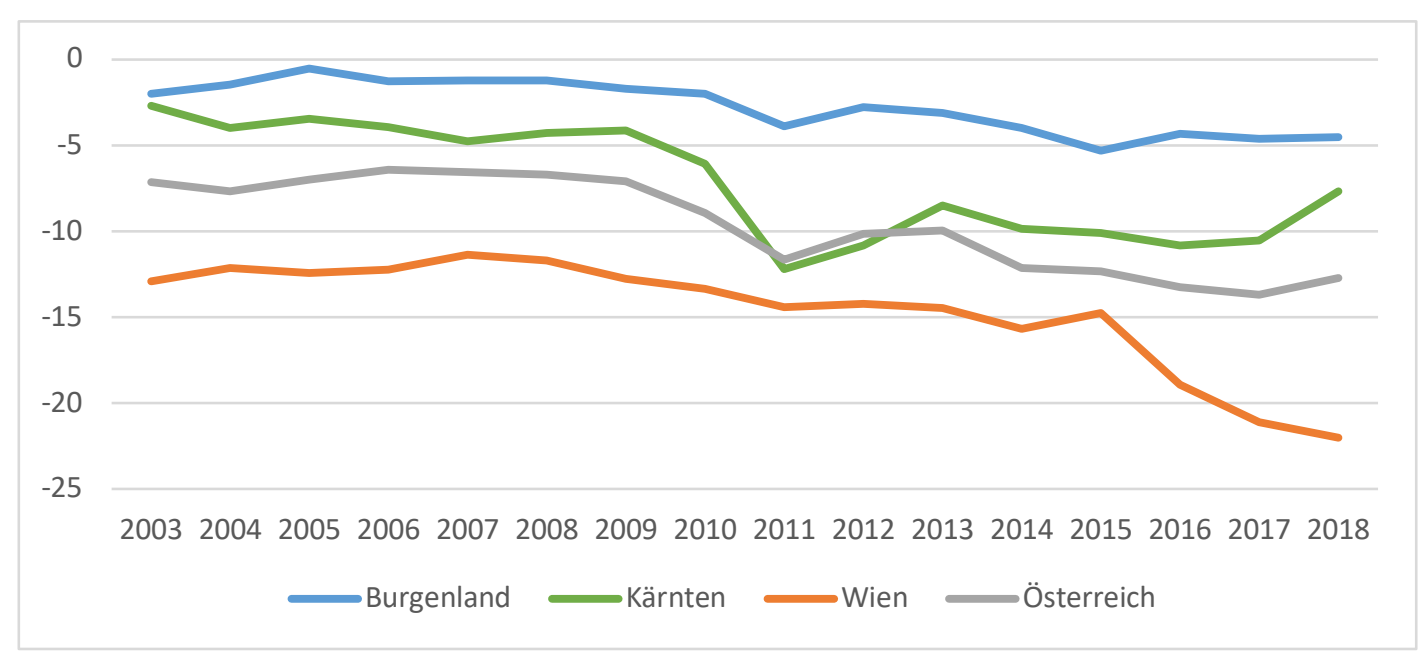

Quelle: Amtsblatt für die evangelische Kirche in Österreich 2004-2019

\section{Schätzung und Projektion der evangelischen Bevölkerung}

Es wurde schon mehrfach versucht, die religiöse Zusammensetzung Österreichs zu schätzen, insbesondere nachdem die Statistik Austria die Frage nach der religiösen Zugehörigkeit aus 
dem Fragebogen der Volkszählung entfernt hatte.5 Anfang der 200oer Jahre war auch die evangelische Kirche daran interessiert zu verstehen, wie sich die zu beobachtenden Trends auf die Zukunft der Mitgliedschaft auswirken könnten. In diesem Zusammenhang prognostizierten Fliegenschnee et al. (2004) die künftigen Mitgliederzahlen der evangelischen Kirche bis 2031, ausgehend von den Volkszählungen von 2001 sowie Informationen über das demografische und mobilitätsbezogene Verhalten (Ein- und Austritte) in der Kirche und mehreren Szenarien über zukünftige Entwicklungen. Diese Schätzungen sind in Tabelle 1 dargestellt.

Tab. 1. Bevölkerungsprognose für Angehörige der evangelischen Kirche in Österreich, Wien, Burgenland und Kärnten, auf der Grundlage von sechs verschiedenen Szenarien, 2001-2031.

\begin{tabular}{|l|l|l|l|l|l|l|}
\hline & $\begin{array}{l}\text { Demo- } \\
\text { graphie }\end{array}$ & Konstant & $\begin{array}{l}\text { Austritte } \\
\text { halbiert }\end{array}$ & $\begin{array}{l}\text { Austritte } \\
\text { verdoppelt }\end{array}$ & $\begin{array}{l}\text { Schwache } \\
\text { Identität }\end{array}$ & $\begin{array}{l}\text { Starke } \\
\text { Identität }\end{array}$ \\
\hline \multicolumn{7}{|c|}{ Österreich } \\
\hline $\mathbf{2 0 0 1}$ & 354.559 & 354.559 & 354.559 & 354.559 & 354.559 & 354.559 \\
\hline $\mathbf{2 0 1 6}$ & 333.945 & $\underline{299.650}$ & 310.228 & $\underline{278.607}$ & 315.752 & 353.225 \\
\hline $\mathbf{2 0 3 1}$ & 306.917 & 245.083 & 274.619 & 191.685 & 271.283 & 346.208 \\
\hline \multicolumn{7}{|c|}{ Wien } \\
\hline $\mathbf{2 0 0 1}$ & 64.085 & 64.085 & 64.085 & 64.085 & 64.085 & 64.085 \\
\hline $\mathbf{2 0 1 6}$ & 60.436 & 49.131 & 52.322 & 42.784 & 57.034 & 61.764 \\
\hline $\mathbf{2 0 3 1}$ & 55.999 & 36.512 & 44.808 & 22.868 & 49.396 & 58.703 \\
\hline & & & Burgenland & & \\
\hline $\mathbf{2 0 0 1}$ & 35.075 & 35.075 & 35.075 & 35.075 & 35.075 & 35.075 \\
\hline $\mathbf{2 0 1 6}$ & 33.069 & 32.669 & 32.883 & 32.249 & 31.247 & 33.780 \\
\hline $\mathbf{2 0 3 1}$ & 30.400 & 29.639 & 30.308 & 28.353 & 26.845 & 31.848 \\
\hline & & & Kärnten & & \\
\hline $\mathbf{2 0 0 1}$ & 56.717 & 56.717 & 56.717 & 56.717 & 56.717 & 56.717 \\
\hline $\mathbf{2 0 1 6}$ & 54.587 & 51.412 & 52.514 & 49.228 & 51.487 & 55.797 \\
\hline $\mathbf{2 0 3 1}$ & 51.300 & 45.351 & 48.580 & 39.306 & 45.175 & 53.798 \\
\hline
\end{tabular}

Quelle: Fliegenschnee et al. (2004).

Anmerkung: Projizierte Mitgliederzahlen, die dem tatsächlichen Wert im Jahr 2016 nahekommen, sind unterstrichen.

Mit 288.328 Mitgliedern im Jahr 2016 (für A.B.) liegt die genaue Zahl der Evangelischen zwischen den Ergebnissen der Projektionen aus den Szenarien „Austritte verdoppelt“ und „konstant“ in Österreich - beide Szenarien sagen auch die Mitglieder in Wien, Burgenland und Kärnten recht gut voraus. Im "konstanten Szenario" wurden konstante Ein- und Austrittsraten auf der Grundlage der Daten um 2001 sowie der Prognosen der Statistik Austria zu Fertilität, Mortalität und Migration angenommen. Eine weitere Annahme war, dass alle Kinder von evangelischen Müttern ebenfalls als Evangelische getauft werden. Im Szenario „Austritte verdoppelt“ sind die demographischen Parameter die gleichen wie im konstanten Szenario,

\footnotetext{
${ }^{5}$ Österreichische Volkszählungen erfassten bis 2001 auch die religiöse Zugehörigkeit der Bevölkerung. Seitdem ist Österreich zu einer komplett registergestützten Volkszählung übergegangen, und auch wenn im Zentralen Melderegister (ZMR) noch Daten über die Religionszugehörigkeit erhoben werden, ist die Angabe der eigenen Religion im Meldezettel nicht obligatorisch, und somit werden keine Daten über die Religionsangehörigkeit mehr veröffentlicht.
} 
jedoch verdoppeln sich die Austrittsraten bis zum Ende des Projektionszeitraums im Jahr 2031. Andere Szenarien wurden wie in Tabelle 1 dargestellt berücksichtigt.

Abb. 4. Anzahl der Evangelischen in Österreich, Schätzungen und Projektionen, 1971-2048

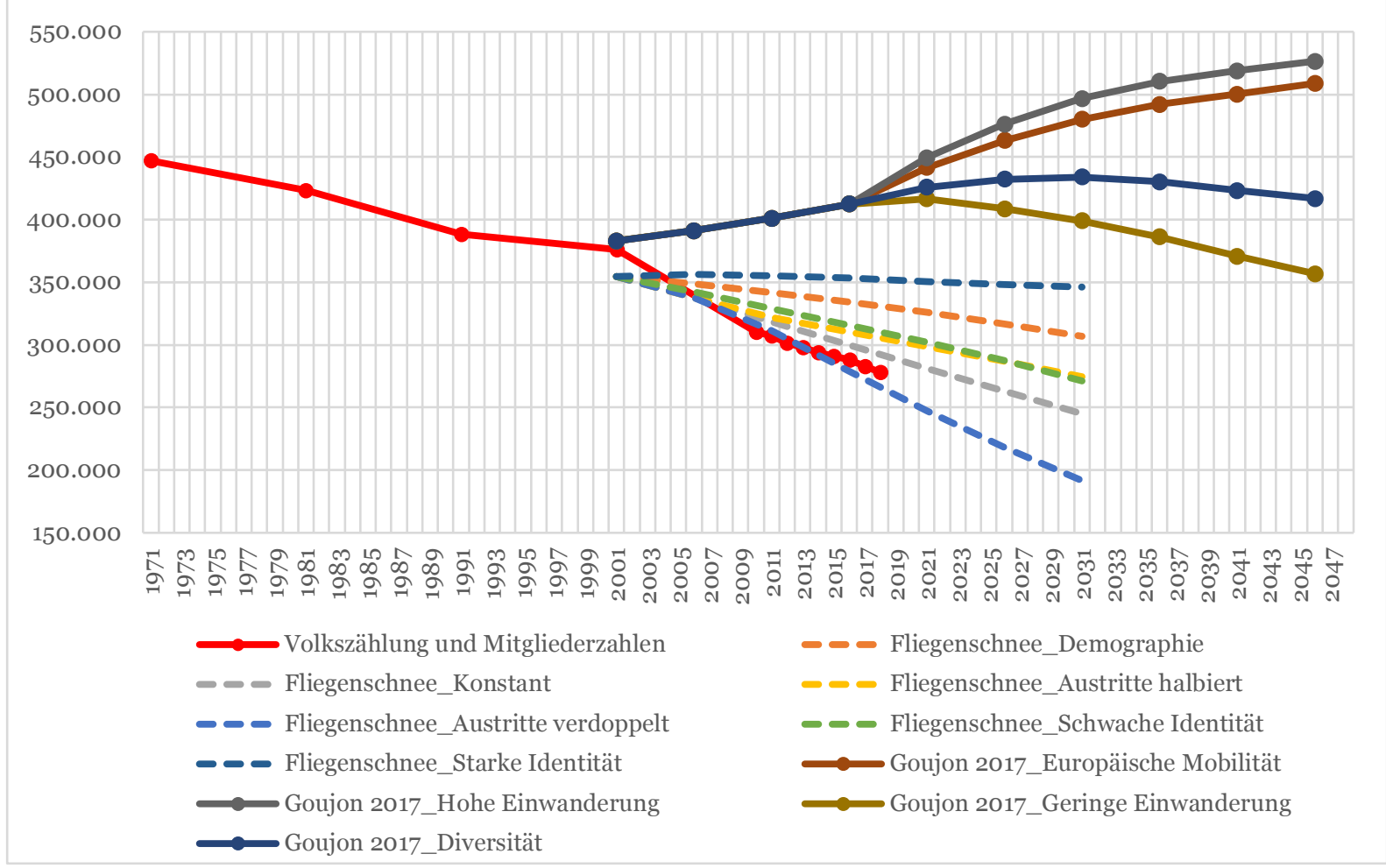

Quellen: 1971-2001: Statistik Austria (Volkszählungen); 2003-2018: Amtsblatt für die evangelische Kirche in Österreich; Fliegenschnee et al. 2004; Goujon et al. 2017 (für den ÖIF/ Österreichischer Integrationsfonds)

Mit dem übergreifenden Ziel, die Veränderungen der religiösen Landschaft Österreichs abzuschätzen, haben einige Studien die Zugehörigkeit zu den verschiedenen Religionen in Österreich (Goujon et al. 2007, 2017), in Wien (Goujon und Bauer 2015; Goujon et al. 2017) und in Vorarlberg (Goujon et al. 2018) rekonstruiert und projiziert. Die Ergebnisse hinsichtlich der Populationsgröße für die evangelische Bevölkerung Österreichs und Wiens sind in Abbildung 4 und 5 dargestellt. Diese Zahlen berücksichtigen auch die tatsächliche Zahl der Kirchenmitglieder von 1971 bis 2018. Alle Prognosen gehen von der Volkszählung 2001 aus dem letzten Mal, als in Österreich die Religionszugehörigkeit erhoben wurde - und rekonstruieren spätere Zahlen für die verschiedenen Religionszugehörigkeiten anhand von Schätzungen und Hypothesen zu Fruchtbarkeit, Migration und religiösen Mobilitätsmustern. Diese Prognosen bieten zwar Schätzungen zum Potenzial der der evangelischen Kirche angeschlossenen Gläubigen, sie unterscheiden sich jedoch von den tatsächlichen Mitgliederzahlen, vor allem weil hier alle protestantischen Zugewanderten, die nach Österreich kommen, als Evangelische gezählt werden, unabhängig davon, ob sie jemals Mitglieder der evangelischen Kirche werden oder nicht - was im Grunde nicht der Fall sein muss. Tatsächlich ist die Zahl der Evangelischen bei diesem Rekonstruktionsverfahren deutlich höher als in der Mitgliederdatenbank der evangelischen Kirche für $2016(+124.000$ für Österreich und +32.00o für Wien), und der Unterschied nimmt mit der Zeit sogar zu, da 
ein großer Teil dieser Zugewanderten höchstwahrscheinlich nicht der evangelischen Kirche beitritt.

Abb. 5. Anzahl der Evangelischen in Wien, Schätzungen und Projektionen

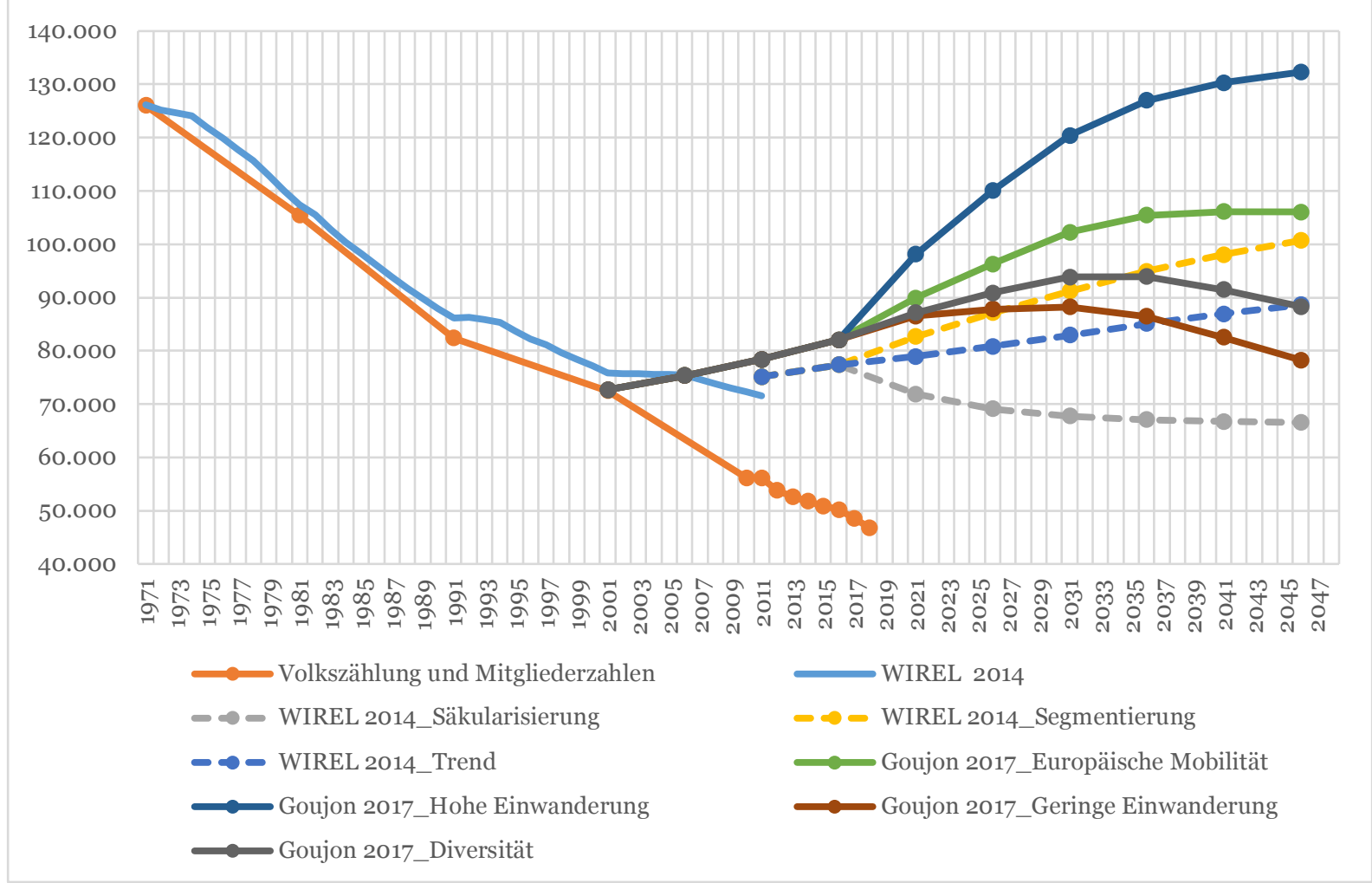

Quellen: 1971-2001: Statistik Austria (Volkszählungen); 2003-2018: Amtsblatt für die evangelische Kirche in Österreich; Goujon et al. 2015; WIREL 2015; Goujon et al. 2017 (für den ÖIF/Österreichischer Integrationsfonds)

Um den Anteil der potenziellen Migrantinnen und Migranten zu ermitteln, die tatsächlich Kirchenmitglieder geworden sind, haben wir die Eintrit von evangelischen Migrantinnen und Migranten in die evangelische Kirche im Zeitraum 2001-2016 simuliert, wobei eine iterative Methode auf Grundlage der Projektionen von Goujon et al. (2017) eingesetzt wurde. Die Ergebnisse zeigen, dass die tatsächliche Veränderung der Mitgliederzahlen zwischen 2001 und 2016 dann am besten mit der Projektion übereinstimmt, wenn man annimmt, dass 30\% der protestantischen Migranten nach Österreich in diesem Zeitraum in die evangelische Kirche eingetreten sind; für Wien beträgt der Anteil, der das gleiche Mitgliedschaftsergebnis wiedergibt, etwa 60\%. Der höhere Anteil im Fall von Wien erklärt sich schlicht dadurch, dass hier viele der „Zugewanderten“ ÖsterreicherInnen sind, die aus einem anderen Bundesland übersiedeln. Diese Ergebnisse werden in den folgenden Hochrechnungen verwendet.

\section{Daten, Methoden und Szenarien}

Um die künftige Entwicklung der evangelischen Kirche in Österreich und in den drei Bundesländern Burgenland, Kärnten und Wien zu prognostizieren, benötigen wir zwei verschiedene Arten von Input: 1) die Basisjahrdaten für die Projektionsparameter Fertilität, Mortalität, Zu- und Abwanderung sowie die Kirchenein- und -austrittszahlen; und 2) 
Szenarien über die mögliche Entwicklung ebendieser Parameter. Der Projektionszeitraum ist jeweils 2018-2048.

\subsection{Basisjahrdaten}

Die Datenbank der Evangelischen Kirche A.B. in Österreich erfasst detaillierte Informationen zu soziodemographischen Merkmalen ihrer Mitglieder. Im Einzelnen enthält sie Daten zu a) Angaben zu Alter, Geschlecht und Gemeinde, b) Sterberegistrierungen von Mitgliedern nach Alter, Geschlecht und Gemeinde und c) Mitgliederzahlen und -bewegungen nach Gemeinde einschließlich Informationen über Ein- und Austritte, Taufen, Todesfälle und internationale $\mathrm{Zu}$ - und Abwanderung. Diese Daten werden jährlich von der evangelischen Kirche erhoben, wobei die letzten vollständigen Daten für das Jahr 2018 vorliegen.

Abbildung 6 zeigt die evangelische Basisjahr-Bevölkerung 2018 nach Alter und Geschlecht für Österreich und die Bundesländer Burgenland, Kärnten und Wien. Während die Gesamtzahl der Evangelischen naturgemäß mit der Bevölkerungsgröße variiert, unterscheidet sich auch der Anteil der evangelischen Angehörigen an der Gesamtbevölkerung in verschiedenen Regionen innerhalb Österreichs deutlich. Mit einem evangelischen Anteil an der Gesamtbevölkerung von 11\% liegt das Burgenland nicht nur im Vergleich zu Gesamtösterreich weit über dem Durchschnitt, es hält auch den höchsten Anteil an Evangelischen unter den drei untersuchten Bundesländern - gefolgt von 8,5\% in Kärnten und nur 2,5\% in Wien. In allen Landesteilen gehören derzeit mehr Frauen als Männer der evangelischen Kirche an.

Abb. 6. Evangelische Bevölkerung (A.B.) nach Alter und Geschlecht, 2018
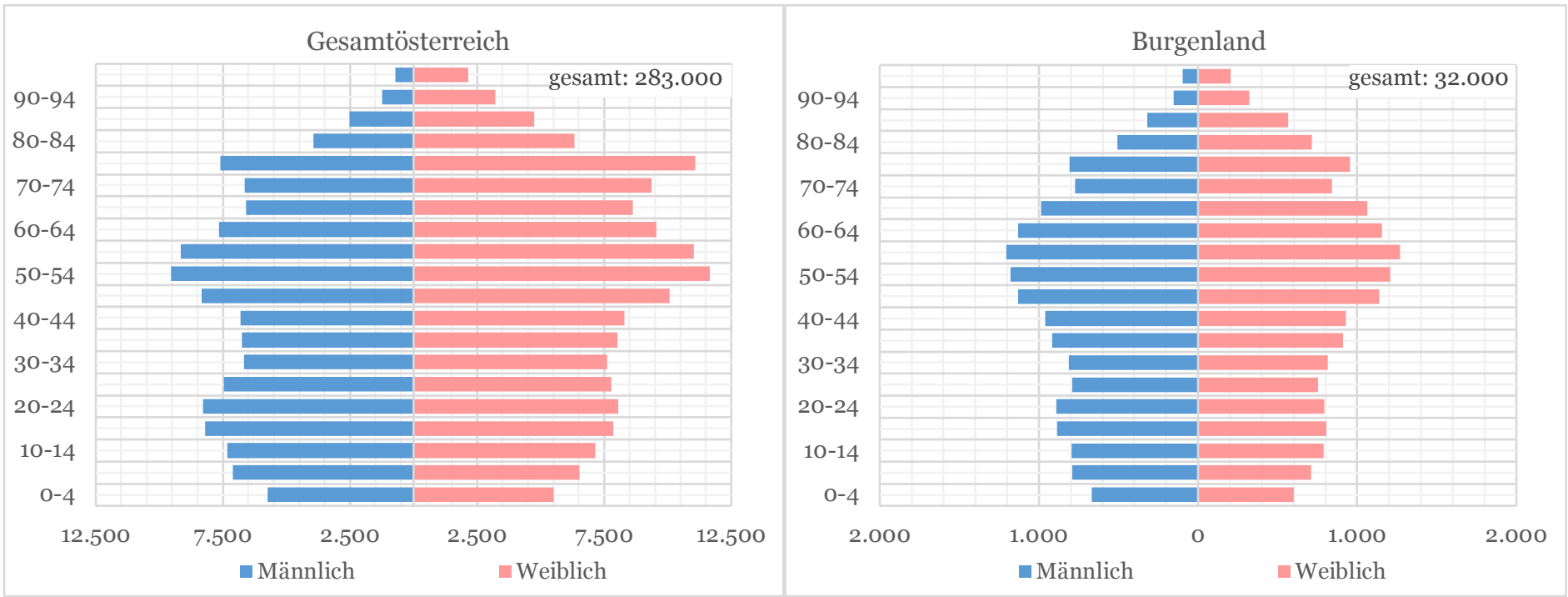


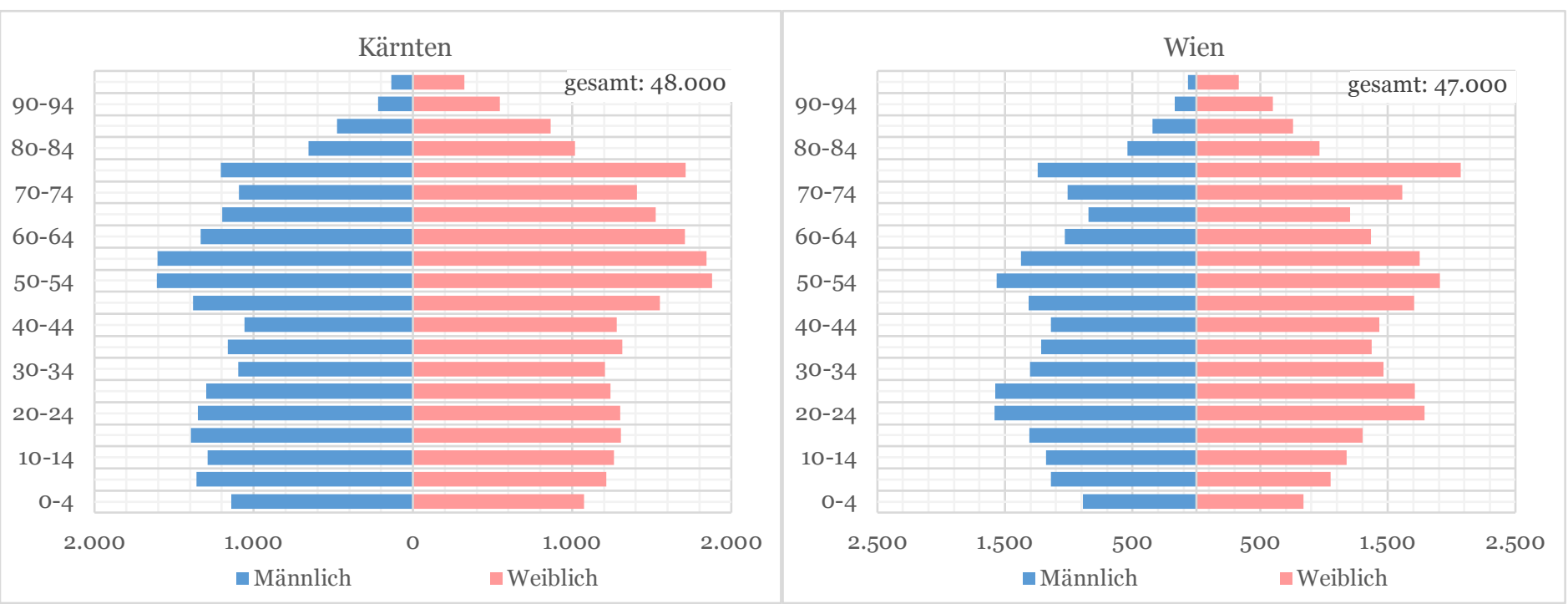

Quelle: Evangelische Kirche A.B.

Anmerkung: Die Abweichung von der in der Grafik dargestellten Gesamtzahl der in Österreich lebenden Angehörigen der A.B. Evangelischen Kirche im Jahr 2018 (283.0oo) zu der oben genannten Zahl (278.ooo) ergibt sich aus unterschiedlichen Datenquellen, die der evangelischen Kirche zugrunde liegen (Seelenstand bzw. Personenabfrage).

Abbildung 7 vergleicht die Altersstruktur der evangelischen Bevölkerung und jene der österreichischen Gesamtbevölkerung (beide Geschlechter zusammengefasst). Wie die Grafik zeigt, ist die evangelische Bevölkerung in den älteren Altersgruppen überproportional vertreten, während die Evangelischen bei den 20- bis 55-Jährigen im Vergleich zu Gesamtösterreich unterrepräsentiert sind. Damit sind die Evangelischen im Durchschnitt älter als die gesamte in Österreich lebende Bevölkerung.

Abb. 7. Vergleich der Altersstruktur zwischen Gesamtbevölkerung und evangelischer Bevölkerung (A.B.), Österreich, 2018

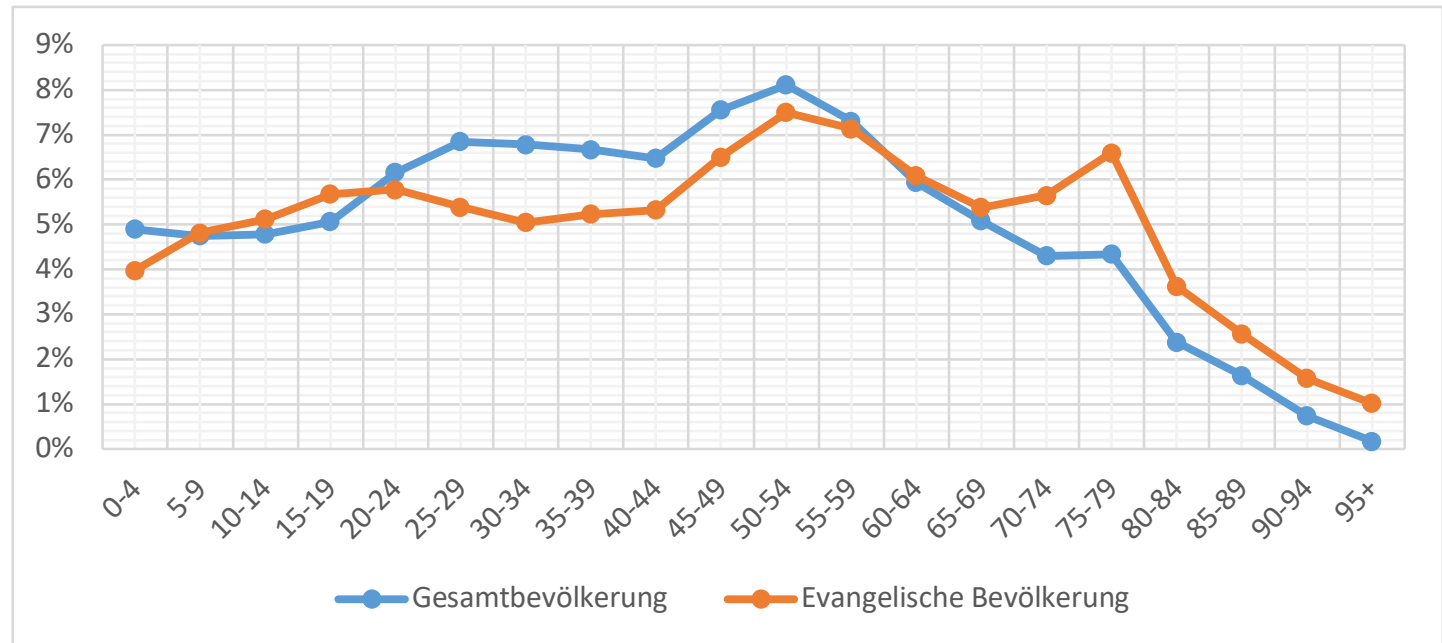

Quellen: Statistik Austria für die Gesamtbevölkerung; Evangelische Kirche A.B. für die evangelische Bevölkerung 
Die Fertilität wird auf Basis von Annahmen aus dem ÖIF-Projekt (Goujon et al. 2017) ${ }^{6}$ geschätzt. In Übereinstimmung mit empirischen Daten, wird die Gesamtfertilitätsrate (total fertility rate/TFR) der evangelischen Frauen im ersten Projektionszeitraum (2018-2023) in allen Bundesländern niedriger angenommen als jene der österreichischen Gesamtbevölkerung, was in den Jahren 2018-2023 eine TFR von durchschnittlich 1,37 Kindern für Gesamtösterreich ergibt (im Vergleich zu 1,54 Kindern der österreichischen Gesamtbevölkerung nach den Hochrechnungen der Statistik Austria (Statistik Austria 2018)). Die Geburtenhäufigkeit der Evangelischen in den drei Bundesländern wurde anteilig bereinigt, so dass sie im Burgenland 1,27 Kinder (im Vergleich zu 1,42), in Kärnten 1,35 Kinder (im Vergleich zu 1,52) und in Wien 1,3 Kinder (im Vergleich zu 1,46) beträgt. Die geringere Fertilität der Evangelischen könnte sich aus ihrem durchschnittlich höheren Bildungsniveau erklären: Nach der Volkszählung von 2001 erreichte der Anteil von Evangelischen mit Hochschulabschluss 14\% gegenüber 10\% für die Gesamtbevölkerung. Umgekehrt hatten 33\% der Protestanten einen Abschluss der unteren Sekundarstufe oder darunter, verglichen mit $39 \%$ in der Gesamtbevölkerung.

Zwar könnte der höhere Bildungsstand der evangelischen Bevölkerung auch die Sterberate senken, doch gingen wir mangels detaillierter Daten zur Schätzung der Überlebenskennzahlen und der Lebenserwartung davon aus, dass Evangelische die gleiche Mortalität wie die übrige Bevölkerung verzeichnen. ${ }^{7}$ Die Lebenserwartung in Österreich variierte im Jahr 2018 zwischen den Bundesländern von 78,2 bis 80,5 Jahren für Männer und zwischen 82,7 und 85 Jahren für Frauen. In Wien lag sie etwas niedriger, im Burgenland und in Kärnten etwas höher.

Die Zahlen zur Migration sind Schätzungen anhand von Daten der Statistik Austria über innerösterreichische und internationale Wanderungsströme sowohl auf Bundes- wie auf Bundesländerebene nach Alter, Geschlecht und Geburtsland. Da über die Religionszugehörigkeit von Zugewanderten keine Informationen vorliegen, wurde angenommen, dass die religiöse Zusammensetzung von Zugewanderten die gleiche wie jene in ihrem Geburtsland ist. So sind etwa 29 Prozent der deutschen Bevölkerung evangelisch; damit werden auch 29 Prozent aller Migrantinnen und Migranten, die mit Deutschland als Geburtsland nach Österreich kommen, als Evangelische gezählt. Wir haben uns hier für das Geburtsland und nicht für die Staatsbürgerschaft als entscheidendes Attribut entschieden, da letztere auch noch nach der Ankunft in einem Land erworben worden sein kann. Darüber hinaus weisen Zugewanderte der ersten Generation, die im Kontext ihres Heimatlandes sozialisiert wurden, in der Regel ein ausgeprägtes demographisches Verhalten auf, das sich in den Daten zur Staatsbürgerschaft verbergen könnte.

Diese Annahme des Zufallsmigranten dürfte die Realität abbilden, es sei denn, die Auswahl von Zugewanderten erfolgt aufgrund ihrer Religion oder anderer eng mit der Religion verbundener Merkmale (z. B. ethnische Zugehörigkeit). Dies könnte insbesondere bei

\footnotetext{
${ }^{6}$ Nach diesen Schätzungen (Goujon et al. 2017) lag die Gesamtfertilitätsrate (TFR) evangelischer Frauen in Österreich 2001-05 bei 1,26 Kindern, 2006-10 bei 1,28 und 2011-2015 bei 1,29, verglichen mit der jeweiligen TFR aller österreichischen Frauen von 1,38, 1,41 und 1,45 Kindern. Bezogen auf Wien bekamen evangelische Frauen 2001-05 durchschnittlich 1,20, 2006-10 1,22 und 2011-15 1,23 Kinder, während alle Frauen in den gleichen Zeiträumen durchschnittlich 1,38, 1,41 und 1,44 Kinder gebaren.

7 Während zwar eine Reihe von Studien das Vorhandensein von Mortalitätsdifferenzen aufgrund des Bildungsniveaus zeigen - Klotz und Doblhammer (2008) wiesen für Männer 6,1 Jahre Unterschied in der Lebenserwartung mit 35 Jahren zwischen Primär- und Tertiärbildung nach, für Frauen 2,8 Jahre -, würde dennoch das beobachtete unterschiedliche Bildungsniveau bei Evangelischen und dem Rest der Bevölkerung keine wesentlichen Schwankung der Langlebigkeit herbeiführen.
} 
Asylsuchenden der Fall sein, da politische Unterdrückung, Diskriminierung, Menschenrechtsverletzungen und gewaltsame Konflikte häufig spezifische Teilpopulationen betreffen, darunter auch religiöse Minderheiten. Da jedoch die meisten der nach Österreich einwandernden Evangelischen aus den Ländern Europas und somit kaum als Flüchtlinge nach Österreich kommen, ist zu erwarten, dass die Annahme des Zufallsmigranten zu realistischen Migrationsschätzungen führt.

Die Anteile der Bevölkerung nach Religion in den jeweiligen Ländern wurden auf zwei Arten ermittelt: für die wichtigsten Länder, aus denen Menschen nach Österreich zuwandern, wurden aktuelle Daten aus der nationalen Statistik (Volkszählungsrunden 2011) erhoben, sofern diese verfügbar waren (Bosnien und Herzegowina, Bulgarien, Kroatien, Tschechische Republik, Estland, Georgien, Deutschland und Schweiz). Für die übrigen Länder basieren die Anteile auf den vom Pew Research Centre veröffentlichten Daten (2011, 2012). Wie Abbildung 8 zeigt, weisen die Anteile der Evangelischen innerhalb einiger der Hauptherkunftsländer von Zugewanderten nach Österreich erhebliche Unterschiede auf.

Abb. 8. Anteile der Evangelischen für einige der Hauptherkunftsländer von Zugewanderten nach Österreich

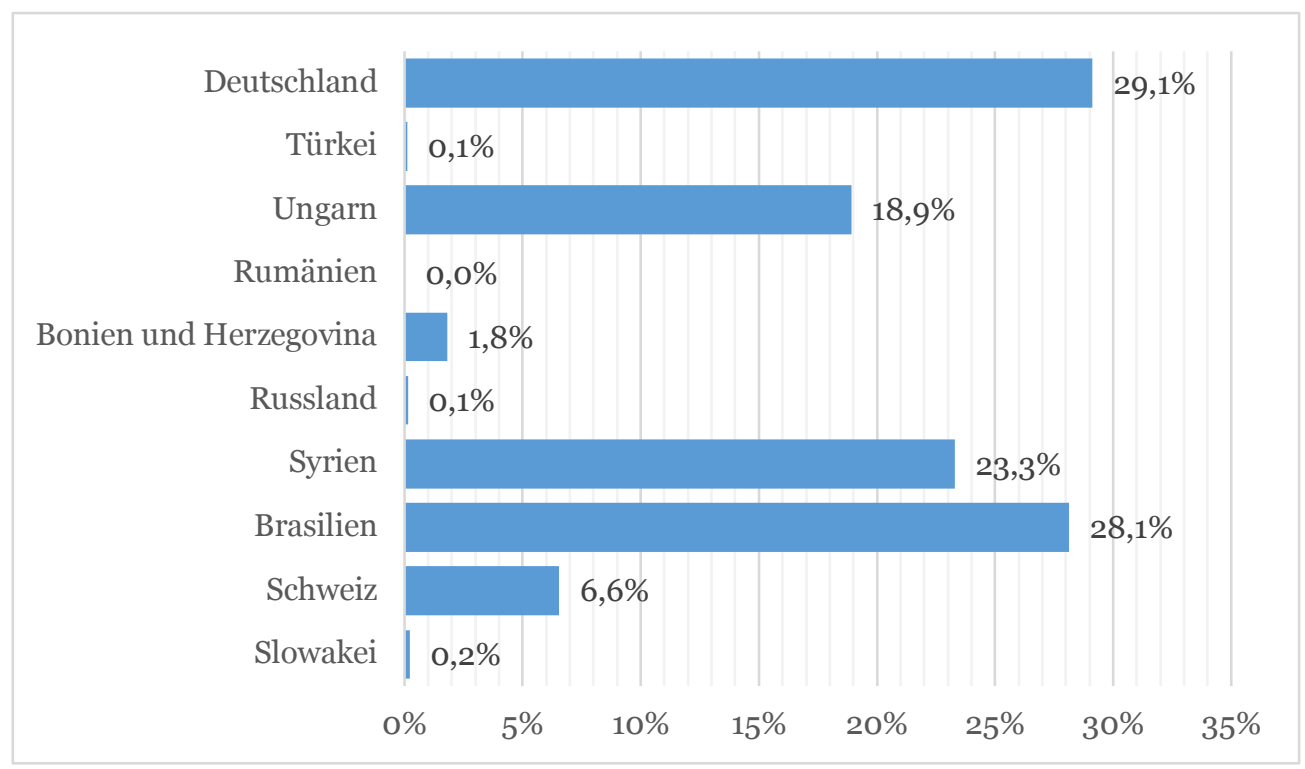

Quelle: Berechnungen der Autorinnen auf Grundlage nationaler Statistiken (2011) und PEW Research Center (2011, 2012)

Die Daten über Ein- und Austritte in die bzw. aus der evangelischen Kirche stammen aus der Datenbank der evangelischen Kirche, die wie oben beschrieben auch Informationen über religiöse Bewegungen auf Gemeindeebene enthält.

\subsection{Diverse Szenarien}

Wir haben fünf Szenarien berechnet, die auf verschiedenen Entwicklungen der letzten Jahre basieren und denen unterschiedliche Narrative zugrunde liegen. Diese Szenarien spiegeln die Sensitivität von Mitgliederzahlen auf unterschiedliche Veränderungen in der Gesellschaft wider, insbesondere betreffend Säkularisierungstrends und Veränderungen in der Zusammensetzung und Mitgliedsbereitschaft von Zugewanderten, die nach Österreich kommen: 
1. Szenario Trend Mitglieder \& Europäische Mobilität (TREND EUROPE): Dabei wird angenommen, dass die demographischen Komponenten dem Trend-Szenario des ÖIFBerichts folgen, der für Österreich einen sehr geringen Anstieg der Fertilität auf 1,58 im Jahr 2048 vorsieht (1,52 in Wien, 1,44 im Burgenland und 1,55 in Kärnten). Wie bereits erwähnt und in Anlehnung an die Analyse von Goujon et al. (2017) ist die Fertilität bei den Evangelischen etwas geringer als im gesamten Land, zudem werden nicht alle Kinder evangelischer Mütter auch evangelisch getauft. Dies spiegelt sich in den Fertilitätsziffern, die an die in den letzten Jahren (2015-18) beobachtete Geburtenzahlen evangelischer Kinder angepasst wurden, wider. Die Lebenserwartung steigt, wie in Tabelle 2 in Anlehnung an die mittlere Variante von Statistik Austria (2018) dargestellt. Auch die Migration folgt der mittleren Variante von Statistik Austria. Wir gehen hier von einer ähnlichen Zusammensetzung aus wie in den Jahren 2006-2010, als die Zuwanderung nach Österreich von Menschen aus Europa dominiert wurde, daher war der Anteil der Evangelischen hier recht hoch (10,6\%). Wir gehen jedoch davon aus, dass nur 30\% aller evangelischen Migrantinnen und Migranten Mitglieder der österreichischen evangelischen Kirche werden (und $60 \%$ aller Nettozuwanderer in die Bundesländer). Diesem Szenario zufolge würde die Nettozahl der Zugewanderten von 23.000 im Zeitraum 2018-22 auf 18.000 im Zeitraum 2042-48 sinken. Die jeweiligen Zahlen für die drei Bundesländer sind in Tabelle 2 dargestellt. Bei den Ein- und Austritten haben wir den in Abbildung 8 dargestellten Trend fortgesetzt. Dies würde zu einem Sinken der Neuzugangszahlen von 728 im Jahr 2018 auf 585 im Jahr 2048 und zu einem Anstieg der Abgänge von 4.468 auf 4.822 führen. Die jeweiligen Zahlen und Werte sind in Abbildung 9 und Tabelle 2 dargestellt.

Abb. 9. Ein- und Austritte in die/aus der evangelischen Kirche, 2010-2018 und Trendlinie

\begin{tabular}{|c|c|}
\hline Eintritte in die evangelische Kirche & Austritte aus der evangelischen Kirche \\
\hline \multicolumn{2}{|c|}{ Österreich } \\
\hline 6.000 & 6.000 \\
\hline 5.000 & 5.000 \\
\hline 4.000 & 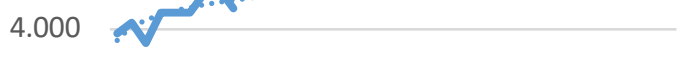 \\
\hline 3.000 & 3.000 \\
\hline 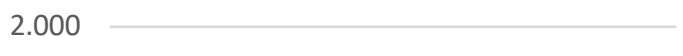 & 2.000 \\
\hline $\mathrm{L}^{\mathrm{n}}$ & 1.000 \\
\hline 0 & 0 \\
\hline $\begin{array}{lllllll}2010 & 2016 & 2022 & 2028 & 2034 & 2040 & 2046\end{array}$ & $\begin{array}{lllllll}2010 & 2016 & 2022 & 2028 & 2034 & 2040 & 2046\end{array}$ \\
\hline
\end{tabular}




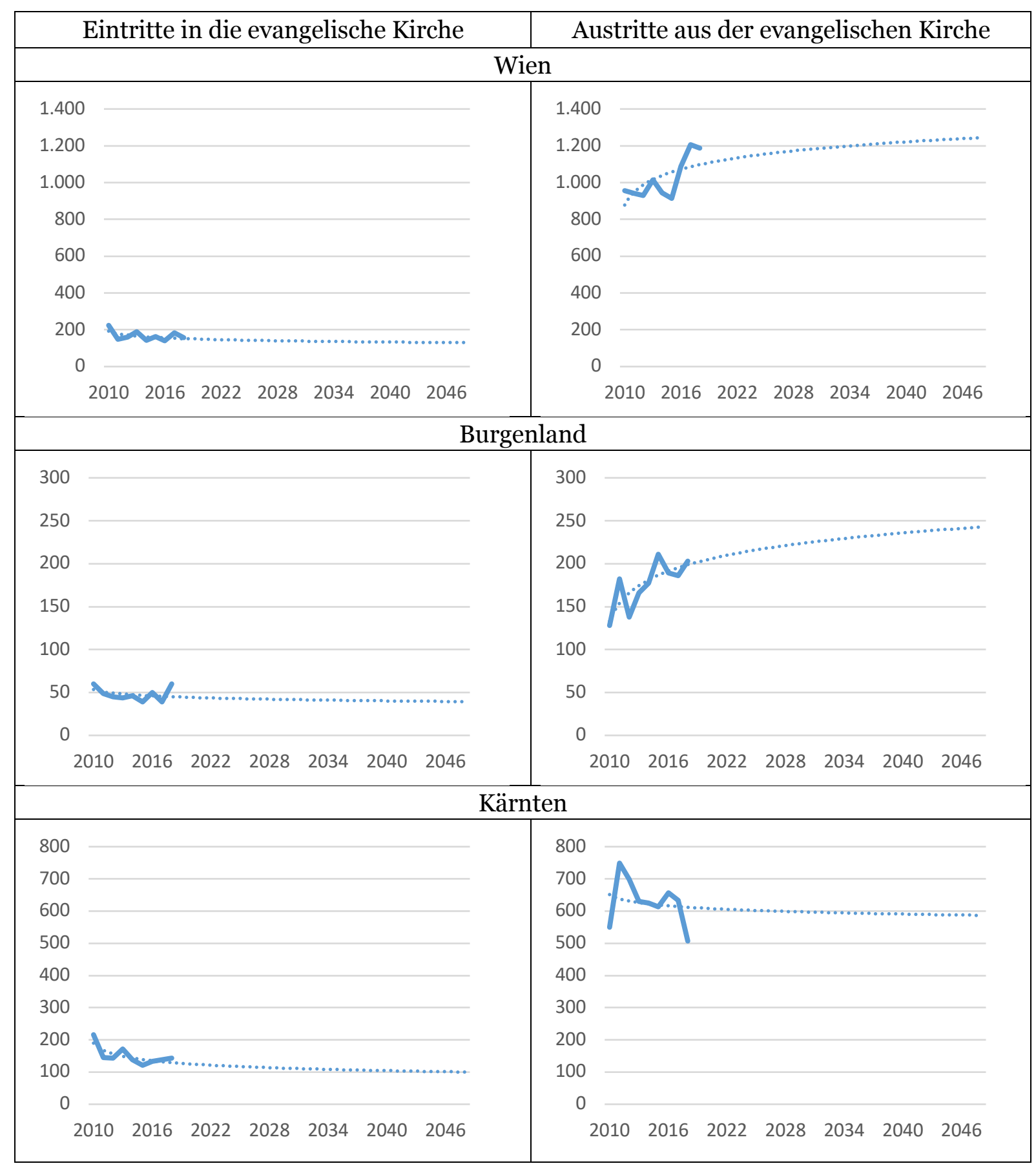

Quelle: Evangelische Kirche (durchgehende Linie) und Berechnungen der Autorinnen (gepunktete Linie)

2. Szenario Trend Mitglieder \& Diversität (TREND DIVERSITY): Dieses Szenario reproduziert fast genau jenes von TREND EUROPE, außer dass die Zusammensetzung der Herkunftsländer von Zugewandertem dem Zeitraum von 2011-15 entspricht, der durch eine verstärkte außereuropäische Komponente gekennzeichnet war, wobei Länder wie Afghanistan, Syrien, Iran und Irak zu den prominentesten Herkunftsländern von Zugewanderten nach Österreich zählten.

3. Szenario Mitgliederanstieg (LIFT): In diesem optimistischen Szenario gehen wir unter Zugrundelegung der Migrationsprämissen des Szenarios TREND EUROPE davon aus, dass mehr Migranten evangelischen Glaubens tatsächlich in die evangelische Kirche eintreten, wobei wir den Eintritt von 60\% der internationalen Migranten für ganz Österreich und von 
80\% für die drei Bundesländer annehmen (im Gegensatz zu den 30\% für Gesamtösterreich und den 60\% für die Bundesländer, die im zuvor beschriebenen TREND EUROPE Szenario angenommen wurden). Auch die Austritte sind um die Hälfte reduziert (die Eintritte folgen dem Szenario TREND_EUROPE). Weiters wird angenommen, dass alle Kinder evangelischer Mütter auch evangelisch getauft werden.

4. Szenario halbierte Austritte \& europäisches Mobilitätsszenario (HALVED EXITS): In diesem Szenario wird untersucht, inwiefern sich ein Rückgang der Säkularisierung auf das TREND Szenario auswirkt. Es werden also Annahmen aus den Szenarien TREND EUROPE und LIFT kombiniert. Fertilitäts- und Migrations-Annahmen sowie die Eintrittszahlen in die evangelische Kirche werden aus dem Szenario TREND EUROPE übernommen. Außerdem wird angenommen, dass sich die Austritte genauso wie im LIFT-Szenario halbieren.

5. Szenario Trend Mitglieder \& Migranten zu Mitgliedern (MIGRANT MEMBER): In diesem Szenario wird untersucht, inwiefern sich eine Zunahme an Mitgliederzahlen von Zugewanderten auf das TREND Szenario auswirkt. Es werden also wiederum Annahmen aus den Szenarien TREND EUROPE und LIFT kombiniert. Fertilitäts-Annahmen sowie die Einund Austritte werden erneut aus dem Szenario TREND EUROPE übernommen. Allerdings geht dieses Szenario davon aus, dass mehr evangelische Zugewanderte in die evangelische Kirche Österreichs eintreten als im LIFT-Szenario.

Die Szenarien 4 und 5 schlüsseln im Wesentlichen den Effekt von Szenario 3 (LIFT) auf. Die Annahmen der Szenarien sind in Tabelle 2 zusammengefasst.

Tab. 2. Annahmen für alle Szenarien, ganz Österreich und drei Bundesländer, für den Zeitraum 2018-2048

\begin{tabular}{|c|c|c|c|c|c|c|}
\hline & TFR & $\begin{array}{l}\text { Lebens- } \\
\text { erwartung } \\
\text { bei Geburt }\end{array}$ & $\begin{array}{l}\text { Durchschn. } \\
\text { Nettozahl } \\
\text { Einwanderung } \\
\text { pro Jahr (A) }\end{array}$ & $\begin{array}{l}\text { Durchschn. } \\
\text { Eintritte } \\
\text { pro Jahr } \\
\text { (B) }\end{array}$ & \begin{tabular}{|l|} 
Durchschn. \\
Austritte \\
pro Jahr \\
(C)
\end{tabular} & $\begin{array}{l}\text { Durchschn. } \\
\text { Netto- } \\
\text { mobilität } \\
\text { pro Jahr } \\
(\text { A+B-C) }\end{array}$ \\
\hline \multicolumn{7}{|c|}{ Österreich } \\
\hline $\begin{array}{l}\text { TREND } \\
\text { EUROPE }\end{array}$ & \multirow{2}{*}{$\begin{array}{l}\text { von } 1,23 \text { bis } \\
1,36 \text { Kinder }\end{array}$} & \multirow{5}{*}{$\begin{array}{l} \\
\text { von } 80,0 \\
\text { bis } 84,5 \\
\text { Jahre für } \\
\text { Männer } \\
\text { und } 84,8 \\
\text { bis } 88,4 \\
\text { Jahre für } \\
\text { Frauen }\end{array}$} & $\begin{array}{l}\text { von }+1404 \text { bis } \\
+1108 \\
\text { Zugewanderte }\end{array}$ & \multirow{5}{*}{$\begin{array}{l}\text { von } 728 \text { bis } \\
585 \\
\text { Eintritte }\end{array}$} & \multirow{2}{*}{$\begin{array}{l}\text { von } 4468 \\
\text { bis } 4822 \\
\text { Austritte }\end{array}$} & \begin{tabular}{|l} 
von \\
-2336 bis \\
-3128 \\
Mitglieder \\
\end{tabular} \\
\hline $\begin{array}{l}\text { TREND } \\
\text { DIVERSITY }\end{array}$ & & & $\begin{array}{l}\text { von }+571 \text { bis } \\
+304 \\
\text { Zugewanderte }\end{array}$ & & & $\begin{array}{l}\text { von } \\
-3169 \text { bis } \\
-3933 \\
\text { Mitglieder } \\
\end{array}$ \\
\hline LIFT & $\begin{array}{l}\text { von } 1,37 \text { bis } \\
1,51 \text { Kinder } \\
\end{array}$ & & $\begin{array}{l}\text { von }+2809 \text { bis } \\
+2217 \\
\text { Zugewanderte } \\
\end{array}$ & & \multirow{2}{*}{$\begin{array}{l}\text { von } 2234 \\
\text { bis } 2410 \\
\text { Austritte }\end{array}$} & \begin{tabular}{|l|} 
von +1303 \\
bis +391 \\
Mitglieder \\
\end{tabular} \\
\hline $\begin{array}{l}\text { HALVED } \\
\text { EXITS }\end{array}$ & \multirow{2}{*}{$\begin{array}{l}\text { von } 1,23 \text { bis } \\
1,36 \text { Kinder }\end{array}$} & & $\begin{array}{l}\text { von }+1404 \text { bis } \\
+1108 \\
\text { Zugewanderte }\end{array}$ & & & $\begin{array}{l}\text { von }-102 \\
\text { bis -717 } \\
\text { Mitglieder }\end{array}$ \\
\hline $\begin{array}{l}\text { MIGRANT } \\
\text { MEMBER }\end{array}$ & & & $\begin{array}{l}\text { von }+2809 \text { bis } \\
+2217 \\
\text { Zugewanderte }\end{array}$ & & $\begin{array}{l}\text { von } 4468 \\
\text { bis } 4822 \\
\text { Austritte }\end{array}$ & $\begin{array}{l}\text { von -931 } \\
\text { bis -2020 } \\
\text { Mitglieder }\end{array}$ \\
\hline
\end{tabular}




\begin{tabular}{|c|c|c|c|c|c|c|}
\hline & TFR & $\begin{array}{l}\text { Lebens- } \\
\text { erwartung } \\
\text { bei Geburt }\end{array}$ & $\begin{array}{l}\text { Durchschn. } \\
\text { Nettozahl } \\
\text { Einwanderung } \\
\text { pro Jahr (A) }\end{array}$ & $\begin{array}{l}\text { Durchschn. } \\
\text { Eintritte } \\
\text { pro Jahr } \\
\text { (B) }\end{array}$ & $\begin{array}{l}\text { Durchschn. } \\
\text { Austritte } \\
\text { pro Jahr } \\
\text { (C) }\end{array}$ & $\begin{array}{l}\text { Durchschn. } \\
\text { Netto- } \\
\text { mobilität } \\
\text { pro Jahr } \\
(\text { A+B-C) }\end{array}$ \\
\hline $\begin{array}{l}\text { TREND } \\
\text { EUROPE }\end{array}$ & \multirow{2}{*}{$\begin{array}{l}\text { von } 1,17 \text { bis } \\
1,31 \text { Kinder }\end{array}$} & \multirow{5}{*}{$\begin{array}{l}\text { von } 78,9 \text { bis } \\
83,7 \text { Jahre } \\
\text { für Männer } \\
\text { und } 83,8 \\
\text { bis } 87,8 \\
\text { Jahre für } \\
\text { Frauen }\end{array}$} & $\begin{array}{l}\text { von }+519 \text { bis } \\
+85 \\
\text { Zugewanderte }\end{array}$ & \multirow{5}{*}{$\begin{array}{l}\text { von } 150 \text { bis } \\
131 \\
\text { Eintritte }\end{array}$} & \multirow{2}{*}{$\begin{array}{l}\text { von } 1135 \\
\text { bis } 1236 \\
\text { Austritte }\end{array}$} & $\begin{array}{l}\text { von } \\
-466 \text { bis } \\
-1021 \\
\text { Mitglieder }\end{array}$ \\
\hline $\begin{array}{l}\text { TREND } \\
\text { DIVERSITY }\end{array}$ & & & $\begin{array}{l}\text { von }+156 \\
\text { bis }-273 \\
\text { Zugewanderte }\end{array}$ & & & \begin{tabular}{|l} 
von \\
-829 bis \\
-1378 \\
Mitglieder
\end{tabular} \\
\hline LIFT & $\begin{array}{l}\text { von } 1.30 \\
\text { bis } 1.45 \\
\text { Kinder }\end{array}$ & & $\begin{array}{l}\text { von }+692 \text { bis } \\
+113 \\
\text { Zugewanderte }\end{array}$ & & \multirow{2}{*}{$\begin{array}{l}\text { von } 567 \text { bis } \\
618 \\
\text { Austritte }\end{array}$} & $\begin{array}{l}\text { von }+274 \\
\text { bis } \\
-374 \\
\text { Mitglieder }\end{array}$ \\
\hline $\begin{array}{l}\text { HALVED } \\
\text { EXITS }\end{array}$ & \multirow{2}{*}{$\begin{array}{l}\text { von } 1.17 \text { bis } \\
1.31 \text { Kinder }\end{array}$} & & $\begin{array}{l}\text { von }+519 \text { bis } \\
+85 \\
\text { Zugewanderte }\end{array}$ & & & $\begin{array}{l}\text { von }+101 \\
\text { bis } \\
-403 \\
\text { Mitglieder }\end{array}$ \\
\hline $\begin{array}{l}\text { MIGRANT } \\
\text { MEMBER }\end{array}$ & & & $\begin{array}{l}\text { von }+692 \text { bis } \\
+113 \\
\text { Zugewanderte }\end{array}$ & & $\begin{array}{l}\text { von } 1135 \\
\text { bis } 1236 \\
\text { Austritte }\end{array}$ & $\begin{array}{l}\text { von } \\
-293 \text { bis } \\
-992 \\
\text { Mitglieder }\end{array}$ \\
\hline \multicolumn{7}{|c|}{ Burgenland } \\
\hline $\begin{array}{l}\text { TREND } \\
\text { EUROPE }\end{array}$ & \multirow{2}{*}{$\begin{array}{l}\text { von } 1.14 \text { bis } \\
1.24 \text { Kinder }\end{array}$} & \multirow{5}{*}{$\begin{array}{l}\text { von } 80.1 \text { bis } \\
84.3 \text { Jahre } \\
\text { für Männer } \\
\text { und } 84.8 \\
\text { bis } 88.2 \\
\text { Jahre für } \\
\text { Frauen }\end{array}$} & $\begin{array}{l}\text { von }+153 \text { bis } \\
+150 \\
\text { Zugewanderte }\end{array}$ & \multirow{5}{*}{$\begin{array}{l}\text { von } 47 \text { bis } \\
40 \text { Eintritte }\end{array}$} & \multirow{2}{*}{$\begin{array}{l}\text { von } 205 \text { bis } \\
240 \\
\text { Austritte }\end{array}$} & $\begin{array}{l}\text { von -5 } \\
\text { bis -51 } \\
\text { Mitglieder }\end{array}$ \\
\hline $\begin{array}{l}\text { TREND } \\
\text { DIVERSITY }\end{array}$ & & & $\begin{array}{l}\text { von +95 bis } \\
\text { +93 } \\
\text { Zugewanderte }\end{array}$ & & & $\begin{array}{l}\text { von -63 bis } \\
-107 \\
\text { Mitglieder }\end{array}$ \\
\hline LIFT & $\begin{array}{l}\text { von } 1.26 \text { bis } \\
1.38 \text { Kinder }\end{array}$ & & $\begin{array}{l}\text { von }+204 \text { bis } \\
+199 \\
\text { Zugewanderte }\end{array}$ & & \multirow{2}{*}{$\begin{array}{l}\text { von } 103 \text { bis } \\
120 \\
\text { Austritte }\end{array}$} & $\begin{array}{l}\text { von }+149 \\
\text { bis }+119 \\
\text { Mitglieder }\end{array}$ \\
\hline $\begin{array}{l}\text { HALVED } \\
\text { EXITS }\end{array}$ & \multirow{2}{*}{\begin{tabular}{|l|} 
\\
von 1.14 bis \\
1.24 Kinder
\end{tabular}} & & $\begin{array}{l}\text { Von }+153 \text { bis } \\
+150 \\
\text { Zugewanderte }\end{array}$ & & & \begin{tabular}{|l|} 
von +98 \\
bis +69 \\
Mitglieder
\end{tabular} \\
\hline $\begin{array}{l}\text { MIGRANT } \\
\text { MEMBER }\end{array}$ & & & $\begin{array}{l}\text { von }+204 \text { bis } \\
+199 \\
\text { Zugewanderte }\end{array}$ & & \begin{tabular}{|l} 
von 205 bis \\
240 \\
Austritte \\
\end{tabular} & $\begin{array}{l}\text { von }+46 \\
\text { bis -1 } \\
\text { Mitglieder }\end{array}$ \\
\hline \multicolumn{7}{|c|}{ Kärnten } \\
\hline $\begin{array}{l}\text { TREND } \\
\text { EUROPE }\end{array}$ & \multirow{2}{*}{$\begin{array}{l}\text { von } 1.22 \text { bis } \\
1.34 \text { Kinder }\end{array}$} & \multirow{3}{*}{$\begin{array}{l}\text { von } 80.1 \text { bis } \\
84.6 \text { Jahre } \\
\text { für Männer } \\
\text { und } 85.2 \text { bis } \\
\text { 88.8 Jahre } \\
\text { für Frauen }\end{array}$} & $\begin{array}{l}\text { von }+196 \text { bis } \\
+237 \\
\text { Zugewanderte }\end{array}$ & \multirow{3}{*}{$\begin{array}{l}\text { von } 128 \text { bis } \\
101 \\
\text { Eintritte } \\
\text { von } 128 \text { bis } \\
101 \\
\text { Eintritte }\end{array}$} & \multirow{2}{*}{$\begin{array}{l}\text { von } 588 \\
\text { bis } 589 \\
\text { Austritte }\end{array}$} & \begin{tabular}{|l} 
von \\
-264 bis \\
-251 \\
Mitglieder
\end{tabular} \\
\hline $\begin{array}{l}\text { TREND } \\
\text { DIVERSITY }\end{array}$ & & & $\begin{array}{l}\text { von }+50 \text { bis } \\
+92 \\
\text { Zugewanderte }\end{array}$ & & & \begin{tabular}{|l} 
von \\
-410 bis \\
-396 \\
Mitglieder \\
\end{tabular} \\
\hline LIFT & $\begin{array}{l}\text { von } 1.35 \text { bis } \\
1.48 \text { Kinder }\end{array}$ & & $\begin{array}{l}\text { von }+261 \text { bis } \\
+316 \\
\text { Zugewanderte }\end{array}$ & & \begin{tabular}{|l|} 
von 294 bis \\
295 \\
Austritte \\
\end{tabular} & \begin{tabular}{|l} 
von +95 \\
bis +123 \\
Mitglieder
\end{tabular} \\
\hline
\end{tabular}




\begin{tabular}{|c|c|c|c|c|c|c|}
\hline & TFR & $\begin{array}{l}\text { Lebens- } \\
\text { erwartung } \\
\text { bei Geburt }\end{array}$ & $\begin{array}{l}\text { Durchschn. } \\
\text { Nettozahl } \\
\text { Einwanderung } \\
\text { pro Jahr (A) }\end{array}$ & $\begin{array}{l}\text { Durchschn. } \\
\text { Eintritte } \\
\text { pro Jahr } \\
\text { (B) }\end{array}$ & $\begin{array}{l}\text { Durchschn. } \\
\text { Austritte } \\
\text { pro Jahr } \\
\text { (C) }\end{array}$ & $\begin{array}{l}\text { Durchschn. } \\
\text { Netto- } \\
\text { mobilität } \\
\text { pro Jahr } \\
(\text { A+B-C) }\end{array}$ \\
\hline $\begin{array}{l}\text { HALVED } \\
\text { EXITS }\end{array}$ & \multirow{2}{*}{$\begin{array}{l}\text { von } 1.22 \text { bis } \\
1.34 \text { Kinder }\end{array}$} & & \multirow{2}{*}{\begin{tabular}{|l|} 
von +196 bis \\
+237 \\
Zugewanderte \\
von +261 bis \\
+316 \\
Zugewanderte \\
\end{tabular}} & & & $\begin{array}{l}\text { von +30 } \\
\text { bis }+44 \\
\text { Mitglieder }\end{array}$ \\
\hline $\begin{array}{l}\text { MIGRANT } \\
\text { MEMBER }\end{array}$ & & & & & $\begin{array}{l}\text { von } 588 \\
\text { bis } 589 \\
\text { Austritte }\end{array}$ & $\begin{array}{l}\text { von -199 } \\
\text { bis -172 } \\
\text { Mitglieder }\end{array}$ \\
\hline
\end{tabular}

Quelle: Hypothesen der Autorinnen, zum Teil basierend auf Goujon et al. (2017)

\section{Ergebnisse}

Die Ergebnisse zeigen, dass die evangelische Kirche in Österreich und in allen Bundesländern außer im Falle des LIFT-Szenarios erhebliche Verluste hinnehmen muss (siehe Abbildungen 10-13). Letzteres geht davon aus, dass die evangelische Kirche durch Zugewanderte und durch die Halbierung der Austritte den Trend der großen Verluste stoppen kann, wodurch sich die Mitgliederzahl für ganz Österreich auf dem Niveau von 2018 mehr oder weniger stabilisieren würde: von 283.000 im Jahr 2018 auf 272.000 im Jahr 2048. Ähnliches gilt für das Burgenland und Kärnten, wo die evangelischen Kirchenmitglieder langsam von 32.00o auf 30.000 (Burgenland) bzw. von 47.000 auf 43.000 (Kärnten) zurückgehen würden. Wien ist das einzige betrachtete Bundesland, wo im LIFT-Szenario die evangelische Bevölkerung leicht ansteigen würde: zwischen 2018 und 2048 von 47.300 auf 47.600. Nach diesem Szenario würde der Anteil der evangelischen Kirchenmitglieder an der Gesamtbevölkerung in Österreich laut der mittleren Prognosevariante der Statistik Austria (2018) von 3,2\% im Jahr 2018 auf 2,8\% im Jahr 2048 zurückgehen.

Um besser verstehen zu können, was diese Änderungen vorantreibt, haben wir dieses Szenario in zwei Szenarien unterteilt, wobei wir den Effekt eines Anstiegs der Mitgliederzahl getrennt betrachten: entweder aufgrund eines Rückgangs der Austritte (Szenario HALVED EXITS) oder eines Zuwachses von Migranten, die der evangelischen Kirche angehören (Szenario MIGRANT MEMBER). Beim Vergleich der beiden Szenarien zeigt sich, dass sich eine Veränderung bei den Austritten stärker auf die Mitgliederzahlen auswirkt: 272.00o Mitglieder im Szenario HALVED EXITS gegenüber 218.0oo Mitgliedern im Szenario MIGRANT MEMBER. Obwohl dies in hohem Maße von dem auf beide Parameter angewandten Korrekturfaktor abhängt, könnte es auch das tatsächlich vorhandene Potenzial der evangelischen Kirche widerspiegeln, ihre Mitglieder an sich zu binden, statt neue Mitglieder anzuziehen, die weniger leicht zugänglich sind.

Alle drei oben beschriebenen Szenarien gehen allerdings von der Umkehr eines in den letzten Jahrzehnten zu beobachtenden Trends aus. Wird diese Entwicklung jedoch beibehalten, wie es die beiden anderen Szenarien zeigen, so wird klar, dass der Rückgang der Anzahl evangelischer Kirchenmitglieder sogar erheblich sein könnte. Das Szenario TREND DIVERSITY würde für die evangelische Kirche bis 2048 landesweit einen Mitgliederverlust von $60 \%$ bedeuten. Die Auswirkungen wären im Burgenland (-32\%) und in Kärnten (-59\%) geringer, in Wien dafür (-65\%) höher. Nach diesem Szenario würde die evangelische Bevölkerung in Österreich am Ende nur 116.000 Mitglieder haben, was $1,2 \%$ der Gesamtbevölkerung entspricht. Die Zahlen für alle Bundesländer sind in Tabelle 1 des Anhangs dargestellt. 
Im Vergleich dazu, bedeutet das Szenario TREND EUROPE einen weniger raschen Rückgang der Mitgliederzahlen, da hier mehr internationale Migranten aus Europa kommen und diese eher protestantisch sind, z.B. wenn sie aus Deutschland stammen. Die evangelische Bevölkerung in Österreich würde von 283.00o im Jahr 2018 auf 144.000 im Jahr 2048 zurückgehen (-49\%). Auf der Ebene der drei Bundesländer fiele der Rückgang in Wien mit 47.000 auf 27.000 ( $-42 \%$ ) deutlicher aus als im Burgenland (-26\%, von 32.000 auf 24.000) und in Kärnten (-37 \%, von 48.000 auf 30.000). Laut diesem Szenario würde die evangelische Bevölkerung Österreichs 1,5 \% der Gesamtbevölkerung ausmachen (1,3 \% in Wien, 7,3 \% im Burgenland und 5,4 \% in Kärnten).

Die Szenarien sind zwar bei weitem nicht als erschöpfend zu betrachten, was die Zukunft der evangelischen Kirche in Österreich betrifft, zeigen aber definitiv die zu erwartenden Tendenzen bei anhaltender bzw. leicht modifizierter Entwicklung der wichtigsten demographischen Faktoren, die sich auf die Mitgliederzahlen in Österreich und in den Bundesländern Burgenland, Kärnten und Wien auswirken. Unter den genannten Annahmen ist in naher Zukunft keine wesentliche Umkehr des rückläufigen Mitgliedertrends zu erwarten. Jedoch könnten unvorhergesehene Änderungen der Parameter zu Entwicklungen bei den Mitgliederzahlen führen, die in keinem der im Rahmen dieses Berichts vorgestellten Szenarien berücksichtigt werden.

Abb. 10. Geschätzte und prognostizierte Zahl der evangelischen Kirchenmitglieder, 20012048, Österreich, alle Szenarien

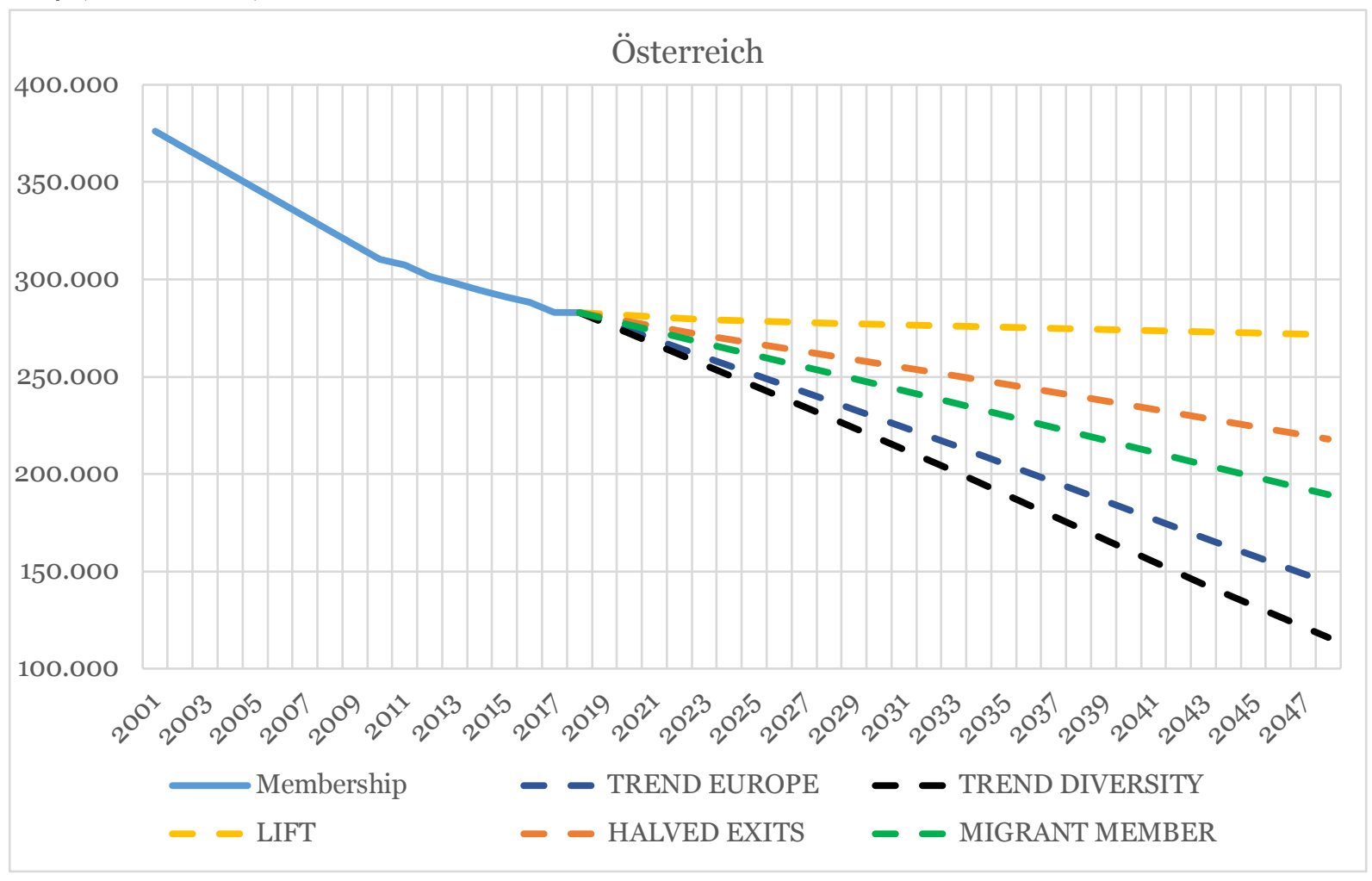

Quelle: Berechnungen der Autorinnen 
Abb. 11. Geschätzte und prognostizierte Zahl der evangelischen Kirchenmitglieder, 20012048, Wien, alle Szenarien

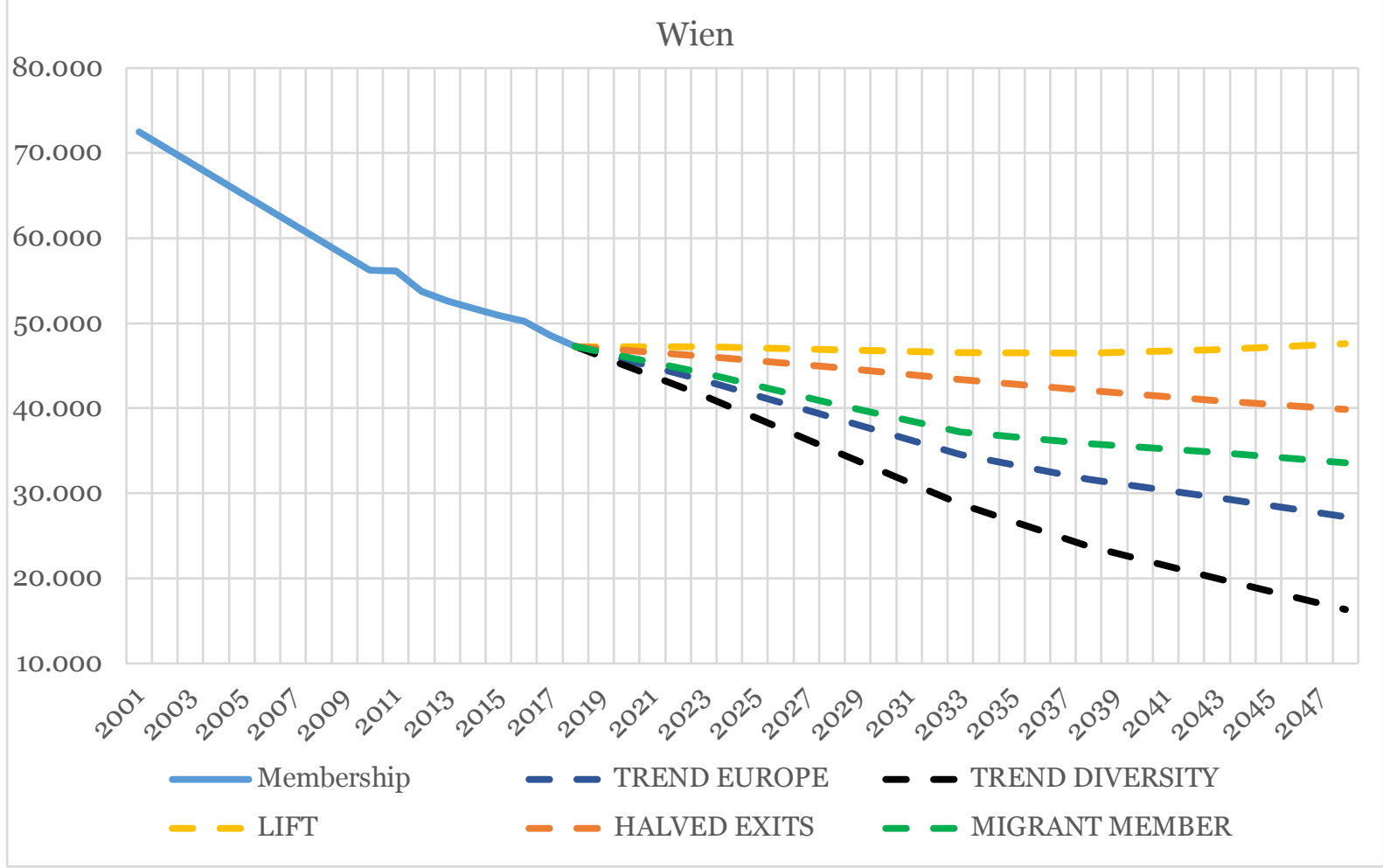

Quelle: Berechnungen der Autorinnen

Abb. 12. Geschätzte und prognostizierte Zahl der evangelischen Kirchenmitglieder, 20012048, Burgenland, alle Szenarien

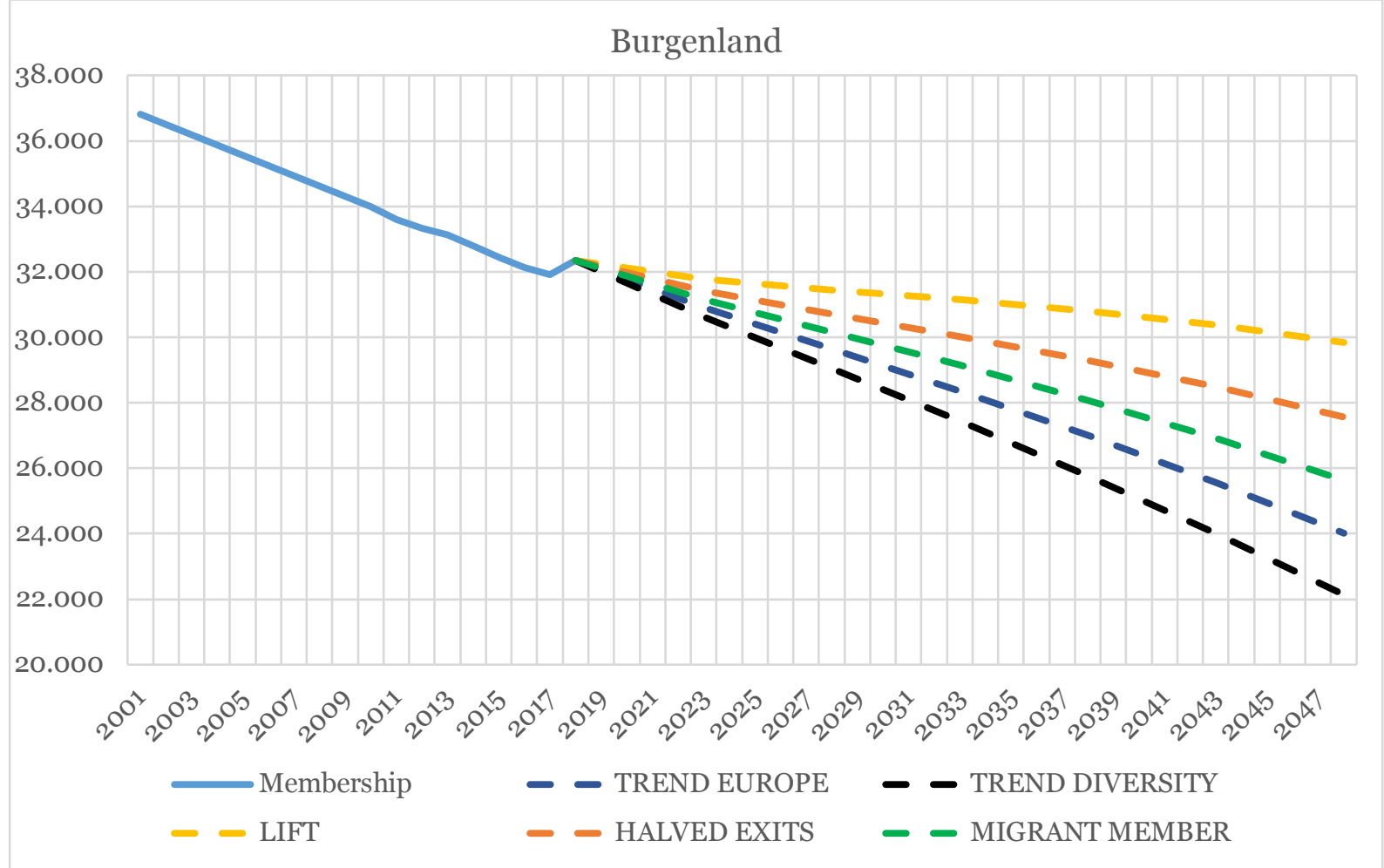

Quelle: Berechnungen der Autorinnen 
Abb. 13. Geschätzte und prognostizierte Zahl der evangelischen Kirchenmitglieder, 20012048, Kärnten, alle Szenarien

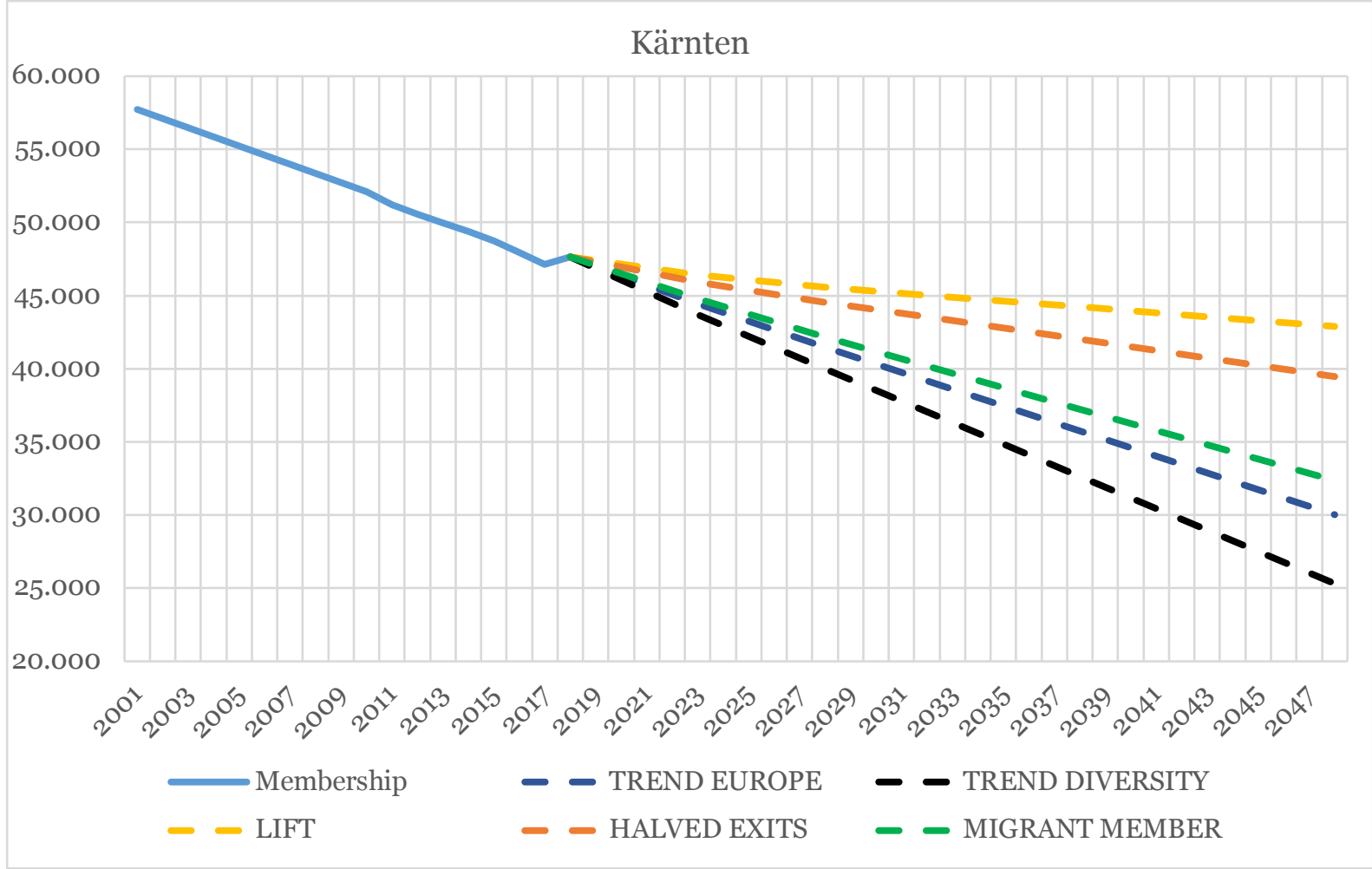

Quelle: Berechnungen der Autorinnen

\section{Schlussfolgerung}

Dieser Bericht hat einige der möglichen Perspektiven aufgezeigt, die sich für die Evangelische Kirche in Österreich und in den Bundesländern Burgenland, Kärnten und Wien durch die möglichen Auswirkungen der Demographie sowie von Kirchenaustritten auf die zukünftigen Mitgliederzahlen in den nächsten Jahrzehnten ergeben könnten. Trotz erheblicher Unterschiede zwischen den Annahmen, die den hier vorgestellten Szenarien zugrunde liegen, deuten fast alle Ergebnisse auf erhebliche Verluste für die evangelische Kirche in Österreich insgesamt wie auch in allen betrachteten Bundesländern hin - wenn auch in unterschiedlichem Ausmaß.

Auch wenn die Szenarien keineswegs erschöpfend sind, was die Zukunftsaussichten für die evangelische Kirche in Österreich angeht, so können doch einige wichtige Schlussfolgerungen hinsichtlich der künftigen Mitgliederzahlen gezogen werden. Erstens sind sowohl in Gesamtösterreich als auch in den drei Bundesländern Burgenland, Kärnten und Wien vergleichbare Entwicklungen zu erwarten, wenn auch in unterschiedlichem Tempo und Ausmaß. Während Wien die größte Variationsbreite zwischen den Szenarien aufweist, die von einem geringen Mitgliederzuwachs im LIFT-Szenario bis zu einem Verlust von zwei Dritteln der Kirchenmitglieder bis 2048 im TREND DIVERSITY-Szenario reicht, sind die Ergebnisse für das Burgenland und Kärnten tendenziell stabiler und weniger sprunghaft. Zweitens: keines der in diesem Bericht entwickelten Szenarien prognostiziert eine echte Trendwende für den Rückgang der Mitgliederzahlen, wie ihn die evangelische Kirche schon bisher erlebt hat. Um sich auf eine Phase des nachhaltigen Mitgliederwachstums auszurichten, wären erhebliche Veränderungen der demographischen Parameter und/oder des (Ab-)Zugehörigkeitsverhaltens 
der evangelischen Bevölkerung notwendig. Schließlich ist neben dem anhaltenden Trend zu vermehrten Kirchenaustritten und abnehmenden Eintritten in die Kirche auch die Herausforderung zu berücksichtigen, einen möglicherweise sehr großen Pool an gläubigen Migrantinnen und Migranten für die evangelische Kirche in Österreich zu gewinnen. 
Anhang Tab. 1. Prognose evangelische Kirchenmitglieder, 2018-2048, Österreich sowie drei Bundesländer

\begin{tabular}{|c|c|c|c|c|c|c|c|}
\hline & 2018 & 2023 & 2028 & 2033 & 2038 & 2043 & 2048 \\
\hline \multicolumn{8}{|c|}{ Österreich } \\
\hline TREND EUROPE & 282.945 & 260.168 & 237.816 & 214.812 & 191.091 & 167.163 & 144.100 \\
\hline $\begin{array}{l}\text { TREND } \\
\text { DIVERSITY }\end{array}$ & 282.945 & 255.999 & 229.167 & 201.334 & 172.537 & $143 \cdot 397$ & 115.998 \\
\hline LIFT & 282.945 & 279.431 & 277.515 & 276.041 & 274.522 & 272.891 & 271.588 \\
\hline HALVED EXITS & 282.945 & 271.338 & 261.020 & 250.661 & 239.876 & 228.865 & 217.927 \\
\hline $\begin{array}{l}\text { MIGRANT } \\
\text { MEMBER }\end{array}$ & 282.945 & 267.187 & 252.073 & 236.679 & 220.868 & 204.914 & 189.423 \\
\hline \multicolumn{8}{|r|}{ Wien } \\
\hline TREND EUROPE & 47.265 & $43 \cdot 312$ & 38.949 & 34.568 & 31.650 & 29.470 & 27.250 \\
\hline $\begin{array}{l}\text { TREND } \\
\text { DIVERSITY }\end{array}$ & 47.265 & 41.503 & 35.076 & 28.715 & $23 \cdot 751$ & 19.972 & 16.348 \\
\hline LIFT & 47.265 & 47.219 & 46.852 & 46.557 & 46.485 & 46.890 & 47.600 \\
\hline HALVED EXITS & 47.265 & 46.147 & 44.800 & 43.406 & 42.089 & 40.855 & 39.870 \\
\hline $\begin{array}{l}\text { MIGRANT } \\
\text { MEMBER }\end{array}$ & 47.265 & 44.179 & 40.543 & 37.246 & 35.826 & 34.853 & 33.592 \\
\hline \multicolumn{8}{|c|}{ Burgenland } \\
\hline TREND EUROPE & 32.343 & 30.902 & 29.633 & 28.347 & 26.996 & 25.561 & 24.015 \\
\hline $\begin{array}{l}\text { TREND } \\
\text { DIVERSITY }\end{array}$ & 32.343 & 30.614 & 29.040 & 27.447 & 25.765 & 24.004 & 22.135 \\
\hline LIFT & 32.343 & 31.776 & 31.442 & 31.153 & 30.806 & 30.368 & 29.840 \\
\hline HALVED EXITS & 32.343 & 31.416 & 30.709 & 30.026 & 29.298 & 28.487 & 27.563 \\
\hline $\begin{array}{l}\text { MIGRANT } \\
\text { MEMBER }\end{array}$ & 32.343 & 31.160 & 30.164 & 29.157 & 28.074 & 26.906 & 25.630 \\
\hline \multicolumn{8}{|r|}{ Kärnten } \\
\hline TREND EUROPE & 47.647 & 44.437 & 41.482 & 38.613 & 35.725 & 32.855 & 30.026 \\
\hline $\begin{array}{l}\text { TREND } \\
\text { DIVERSITY }\end{array}$ & 47.647 & 43.711 & 39.997 & 36.338 & 32.647 & 28.969 & $25 \cdot 316$ \\
\hline LIFT & 47.647 & 46.405 & 45.579 & 44.909 & 44.236 & 43.561 & 42.896 \\
\hline HALVED EXITS & 47.647 & 45.909 & 44.531 & 43.281 & 42.022 & 40.754 & 39.469 \\
\hline $\begin{array}{l}\text { MIGRANT } \\
\text { MEMBER }\end{array}$ & 47.647 & 44.765 & 42.188 & 39.717 & 37.239 & 34.780 & 32.366 \\
\hline
\end{tabular}

Quelle: Evangelische Kirche sowie Berechnungen der Autorinnen 


\section{Literatur}

Buber-Ennser, I., Goujon, A., Kohlenberger, J., Rengs, B. 2018. Multi-Layered Roles of Religion among Refugees Arriving in Austria around 2015. Religions 2018, 9(5), 154.

Fliegenschnee, K., Goujon, A., Lutz, W. 2004. Neue demographische Szenarien zur Zukunft der Evangelischen Kirche in Österreich. VID Working Paper 01/2004. Vienna: VID, Austrian Academy of Sciences.

Goujon, A. und Bauer, R. 2015. Demographic forces shaping the religious landscape of Vienna, Chapter 9.3, Volume 4. In Brunn, S.D. (Ed), The Changing World Religion Map. Springer Netherlands: $2145-2161$.

Goujon, A., Jurasszovich, S. Potančoková, M. 2017. Demographie und Religion in Österreich: Szenarien 2016 bis 2046. ÖIF-Forschungsbericht.

Goujon, A., Skirbekk, V., Fliegenschnee, K., Strzelecki, P. 2006. New Times, Old Beliefs: Projecting the Future Size of Religions in Austria. VID Working Paper 01/2006. Vienna Institute of Demography, Austrian Academy of Sciences. Vienna, 38 pp.

Goujon, A., Skirbekk, V., Fliegenschnee, K., Strzelecki, P. 2007. New Times, Old Beliefs: Projecting the Future Size of Religions in Austria. 2007 Vienna Yearbook of Population Research, 237-270.

Klotz J., Doblhammer, G. 2008. Trends in educational mortality differentials in Austria between 1981/82 and 2001/2002: A study based on a linkage of census data and death certificates. Demographic Research, 19: 1759-1780.

Potančoková, M., Jurrasszovich, S., Goujon, A. 2018. Consequences of international migration on the size and composition of religious groups in Austria. Journal for international migration and integration 19(4): 905-924.

Statistik Austria. 2018. Ergebnisse der Bevölkerungsprognose 2018. Vienna: Statistik Austria. Download auf:

http://www.statistik.at/web_de/statistiken/menschen_und gesellschaft/bevoelkerung/ demographische prognosen/bevoelkerungsprognosen/index.html (Zugriff am 20.10.2019)

WIREL. 2015. Religions in Vienna in the Past, Present and Future - Key Findings from the WIREL Project. WIREL Project, Vienna Institute of Demography, Vienna, Austria. 